\title{
Formation of the first stars and black holes
}

\author{
L. Haemmerlé, L. Mayer, R. S. Klessen, \\ T. Hosokawa, P. Madau \& V. Bromm
}

Received: date / Accepted: date

\begin{abstract}
Keywords
Abstract We review the current status of knowledge concerning the early phases of star formation during cosmic dawn. This includes the first generations of stars forming in the lowest mass dark matter halos in which cooling and condensation of gas with primordial composition is possible at very high redshift $(z>20)$, namely metal-free Population III stars, and the first generation of massive black holes forming at such early epochs, the socalled black hole seeds. The formation of black hole seeds as end states of the collapse of Population III stars, or via direct collapse scenarios, is discussed. In particular, special emphasis is given to the physics of supermassive stars as potential precursors of direct collapse black holes, in light of recent results of stellar evolution models, and of numerical simulations of the early stages of galaxy formation. Furthermore, we discuss the role of the cosmic radiation produced by the early generation of stars and black holes at high redshift in the process of reionization.
\end{abstract}

\section{First stars}

The first generation of stars, the so-called Population III (or Pop. III) built up from truly metal-free primordial gas. They have long been thought to live short, solitary lives, with only one extremely massive star forming in each dark matter halo with about 100 solar masses or more (Omukai, 2001. Abel et al., 2002, Bromm et al., 2002; O'Shea and Norman, 2007). The idea was that first star formation is simple and one only needs to know the initial Gaussian density perturbations of material at very high-redshift which are very well understood, e.g. from measuring cosmic microwave background (CMB) anisotropies (Planck Collaboration et al., 2016a), the growth of cosmological structures, and the heating and cooling processes in the primordial gas. However, this simple picture has undergone substantial 
revision in recent years, and we now understand that stellar birth in the early Universe is subject to similar complexity as star formation at present days. Numerical simulations indicate that fragmentation is a wide-spread phenomenon in first star formation (Clark et al., 2011a; Greif et al., 2012), and consequently that Pop. III stars form as members of multiple stellar systems with separations as small as the distance between the Earth and the Sun (e.g. Turk et al., 2009, Clark et al., 2011b; Greif et al., 2011; Smith et al., 2011; Stacy and Bromm, 2013). Studies that do include radiative feedback (Hirano et al., 2014, 2015; Hosokawa et al., 2016), magnetic fields (Machida et al., 2006, 2008 Schleicher et al., 2009, 2010, Sur et al. 2010, 2012 Turk et al. 2012; Schober et al., 2012b a; Bovino et al., 2013), dark matter annihilation (Smith et al., 2012; Stacy et al., 2014), as well as the primordial streaming velocities (Tseliakhovich and Hirata, 2010; Greif et al., 2011; Maio et al., 2011a; Stacy et al., 2011) add further levels of complexity. It turns out that all of these processes are relevant and need to be incorporated in our theoretical models. There is agreement now that primordial star formation is equally dynamic and difficult to understand as stellar birth at present days (e.g. Mac Low and Klessen, 2004; McKee and Ostriker, 2007; Klessen and Glover, 2016)

In this section we investigate how star formation progresses once the combination of gravitational collapse and cooling leads to a strong accretion flow into the center of a high-redshift halo, and we discuss the most important physical processes that govern stellar birth on small scales. We focus on the standard pathway to Pop. III star formation. We argue that the accretion disk that forms around the first object is likely to fragment, which typically results in the formation of a cluster of stars with a wide range of masses rather than the built-up of a single high-mass object. We then speculate about how stellar feedback, the presence of magnetic fields, the potential energy input from dark matter annihilation and the possible existence of large-scale streaming velocities between baryons and dark matter may influence this picture. Supermassive stars and the possible seeds for supermassive black holes will be discussed in Sections 2 and 3. respectively. The implications on the reionization of the Universe are addressed in Sect. 4

\subsection{Spherical collapse models}

The simplest model for primordial star formation is the run-away collapse of a spherically symmetric halo with mass exceeding the critical value for the cooling instability to set in (Glover, 2005, 2013). This was studied extensively in the early 2000's (Abel et al., 2002 Bromm et al., 2002, Bromm and Larson, 2004, Yoshida et al., 2003, 2006, 2008). Figure 1 adopted from Clark et al. (2011b) shows a typical example of such a system. And from the numbers provided, we can obtain a crude estimate of the accretion rate onto the center of the halo as

$$
\dot{M}=\zeta \frac{M_{\mathrm{J}}}{\tau_{\mathrm{ff}}} \propto \frac{c_{\mathrm{s}}^{3}}{G},
$$


where $M_{\mathrm{J}} \approx 50 \mathrm{M}_{\odot} \mu^{-2} n^{-1 / 2} T^{3 / 2}$ is the Jeans mass with number density $n$ measured in particles per cubic centimeter, temperature $T$ in Kelvin, and mean molecular weight $\mu$ being $\sim 1.22$ for primordial atomic and $\sim 2.33$ for primordial molecular gas, where $\tau_{\mathrm{ff}} \approx 52 \mathrm{Myr} \mu^{-1 / 2} \mathrm{n}^{-1 / 2}$ is the freefall time, and where $c_{\mathrm{s}}=\left(k_{\mathrm{B}} T / \mu m_{\mathrm{H}}\right)^{1 / 2}$ is the sound speed with the Boltzmann constant $k_{\mathrm{B}}$ and the proton mass $m_{\mathrm{H}}$. Because of $\dot{M} \propto T^{3}$, warmer gas leads to higher accretion rates. The factor $\zeta$ can take values of up to several tens depending on the actual density profile and by how much the actuall gas mass $M$ exceeds the Jeans mass $M_{\mathrm{J}}$ (for classical studies, see Shu, 1977; Larson, 1969, Penston, 1969, Whitworth and Summers, 1985). With a typical gas temperature of $T \sim 1500 \mathrm{~K}$ we get accretion rates of $\dot{M} \approx 2 \times 10^{-3} \mathrm{M}_{\odot} \mathrm{yr}^{-1}$.

[Fig. 1 about here.]

\subsection{Disk fragmentation}

These early calculations typically stopped when the object in the center reached number densities of $n \approx 10^{16} \mathrm{~cm}^{-3}$ or more, because then the computational timestep became prohibitively small. At this time the protostar has a mass of only $\sim 10^{-3} \mathrm{M}_{\odot}$. However, as more and more gas falls to the center it also carries along more and more angular momentum. Eventually this gas settles into a rotationally supported disk rather than being consumed by the central protostar. Theoretical and numerical models that follow the build-up and longer-term evolution of this disk show that the system is prone to fragmentation.

Disk fragmentation on various spatial and temporal scales is reported by various authors (Machida et al., 2008; Clark et al., 2011b; Greif et al. 2011, 2012 : Dopcke et al., 2013; Susa|, 2013; Susa et al., 2014; Hosokawa et al., 2016; Stacy and Bromm, 2013; Stacy et al., 2016; Turk et al., 2009; Wollenberg et al. (2020). Under typical conditions of Pop. III star formation the mass load onto the disk by accretion from the infalling envelope exceeds its capability to transport this material inwards by gravitational or magnetoviscous torques, that is by spiral arms (Binney and Tremaine, 1987) or by the magnetorotational instability (Balbus and Hawley, 1998). Consequently the disk becomes Toomre unstable and fragments. This preferentially occurs at the outer edge of the disk. The instability region moves outwards as the disk grows larger by accretion of higher angular momentum material, and the formation of new protostars occurs at larger and larger radii as the evolution progresses. This is indicated in Figure 1. adopted from Clark et al. (2011b), which shows the build-up of altogether four protostars within only about hundred years after the formation of the first object.

Disk fragmentation has important consequences for the resulting stellar mass spectrum. As matter flows through the disk towards the center, it first encounters the Hill volume of secondary protostars further out and preferentially gets swallowed by these objects. Otherwise, this gas would have 
continued to move inwards and would eventually be accreted by the central object. Clearly, disk fragmentation limits the mass growth of the primary star in the center, and so this process has been termed 'fragmentationinduced starvation' in the context of present-day star formation (Kratter and Matzner, 2006; Peters et al., 2010; Girichidis et al., 2011, 2012a b). Some of these protostars may get ejected by dynamical encounters with other protostars or fragments while some may move inwards to get accreted by the central object (Clark et al., 2011a; Greif et al., 2012, Smith et al. 2012, Stacy et al. 2016). As a consequence of these stochastic behavior, the mass spectrum of Pop. III stars is expected be very broad, possibly reaching down into the substellar regime. We conclude that the standard pathway of Pop. III star formation leads to a stellar cluster with a wide distribution of masses rather than the build-up of one single high-mass object.

\subsection{Radiative feedback}

Massive Pop III stars are expected to enter the hydrogen burning main sequence while still accreting (Zinnecker and Yorke, 2007; Maeder, 2009). For accretion rates below $\dot{M} \approx 10^{-3} \mathrm{M}_{\odot}$ they are thought to be compact and very hot at their surface (Hosokawa and Omukai, 2009a; Hosokawa et al. 2010,2012 ), implying that they emit large numbers of ionizing photons (Schaerer, 2002) that can significantly influence their birth environment. For rates much larger than $\dot{M} \gtrsim 10^{-2} \mathrm{M}_{\odot}$ stellar evolution calculations suggest that stars remains bloated and fluffy (Hosokawa et al., 2013; Umeda et al., 2016; Woods et al., 2017; Haemmerlé et al., 2018b). In this case, the surface temperature is relatively low, and although very luminous, these stars do not emit much ionizing radiation and could be able to maintain this high accretion flow for a very long time. In the right environment this could possibly lead to the formation of supermassive stars as we discuss in Section 2 .

Compact and hot Pop. III stars create HII regions which eventually break out of the parental halo and may affect stellar birth in neighboring systems (see, e.g. Kitayama et al., 2004, Whalen et al., 2004; Alvarez et al., 2006, Abel et al., 2007; Yoshida et al., 2007; Greif et al., 2008, Wise et al. 2012b a: Jeon et al. 2014). The question is when this happens and how radiative feedback influences the immediate birth environment. When addressing this problem, we can seek guidance from models of stellar birth at present days. Recent simulations (Yorke and Sonnhalter. 2002. Krumholz et al., 2009; Kuiper et al., 2010, 2011; Peters et al., 2010, 2011 Commerçon et al. | 2011: Rosen et al. 2016) indicate that once a protostellar accretion disk has formed, it quickly becomes gravitationally unstable and so material in the disk midplane flows inwards along dense filaments, whereas radiation escapes through optically thin channels above the disk. Even ionized material can be accreted, if the accretion flow is strong enough (Keto, 2007, Peters et al., 2010). Radiative feedback is found not to be able to shut off the accretion flow, instead it is the dynamical evolution of the disk material that controls the mass growth of individual protostars. 
Due to the lack of metals and dust, protostellar accretion disks around Pop. III stars can cool less efficiently and are much hotter than disks at present days (Tan and McKee, 2004). Furthermore, the stellar radiation field couples less efficiently to the surrounding because the opacities are smaller. It is thus not clear how well the above results can be transferred to the primordial case. Numerical simulations in two dimensions by Hosokawa et al. (2011a), Hirano et al. (2014) and Hirano et al. (2015) find that radiative feedback can indeed stop stellar mass growth and quickly remove the remaining accretion disk, resulting in final stellar masses from a few $10 \mathrm{M}_{\odot}$ up to about $1000 \mathrm{M}_{\odot}$. However, these calculations cannot capture disk fragmentation and the formation of multiple stellar systems. Threedimensional calculations by Stacy et al. (2012), Susa (2013, 2014), or Hosokawa et al. (2016) on the other hand find widespread fragmentation with a wide range of stellar masses down to $\sim 1 \mathrm{M}_{\odot}$. All calculations have limitations and shortcomings, either in terms of resolution or in the number of physical processes included. High resolution is essential for correctly computing the recombination rate in ionized or partially ionized gas. It goes quadratic with the density, and consequently, not resolving the highdensity shell of gas swept up by the expanding ionized gas can result in incorrect HII region expansion rates and sizes (see, e.g. Rahner et al., 2017 2019). By a similar token, treating only ionizing radiation and neglecting the impact of Lyman-Werner wavebands can lead to large errors in the overall photon escape fraction (Schauer et al. 2015$)$. Consequently, any firm conclusions about the resulting mass spectrum of Pop. III stars in the presence of radiative feedback seems premature at this stage.

\subsection{Magnetic fields}

Also the presence of dynamically important magnetic fields will influence disk evolution and fragmentation. Unfortunately, we know very little about magnetic fields in the high-redshift universe. Theoretical models predict that magnetic fields could be produced in various ways, for example via the Biermann battery (Biermann, 1950), the Weibel instability (Lazar et al. 2009 Medvedev et al. 2004), or thermal plasma fluctuations (Schlickeiser and Shukla, 2003). Other models invoke phase transitions that occur during cosmic inflation (Sigl et al., 1997; Grasso and Rubinstein, 2001; Banerjee and Jedamzik, 2003, Widrow et al., 2012). In each case, the inferred field strengths are very small. The only way to obtain dynamically significant magnetic fields is through the small-scale turbulent dynamo, which is very efficient in amplifying even extremely small primordial seed fields to the saturation level. This process is very fast and acts on timescales much shorter than the dynamical free-fall time. An analytic treatment is possible in terms of the Kazantsev model (Kazantsev, 1968; Subramanian, 1998 Schober et al., 2012b a). Once backreactions become important, the growth rate slows down, and saturation is reached within a few large-scale eddyturnover times (Schekochihin et al. 2002, 2004, Schober et al., 2015).

Depending on the properties of the turbulent flow, the magnetic energy density at saturation is thought to lie between $0.1 \%$ and a few $10 \%$ of 
the kinetic energy density (Federrath et al., 2011, 2014). Fields this strong affect the evolution of protostellar accretion disks: They remove angular momentum from the star-forming gas (Machida et al., 2008; Machida and Doi 2013; Bovino et al., 2013; Latif et al., 2013b, 2014b), drive protostellar jets and outflows (Machida et al., 2006), and they reduce the level of fragmentation in the disk (Turk et al. 2011; Peters et al., 2014). Altogether, we expect Pop. III clusters to have fewer stars with higher masses than predicted by purely hydrodynamic simulations. However, a full assessment of these differences still needs to be worked out in detail.

\subsection{Streaming velocity}

Prior to recombination, baryons are tightly coupled to photons resulting in a standing acoustic wave pattern (Sunyaev and Zel'dovich, 1970). This results in oscillations between baryons and dark matter with relative velocities of about $30 \mathrm{~km} \mathrm{~s}^{-1}$ and coherence lengths of several $10 \mathrm{Mpc}$ to $100 \mathrm{Mpc}$ (Silk. 1986 ) at $z \approx 1000$. After recombination, baryons are no longer tied to photons, their sound speed drops to $\sim 6 \mathrm{~km} \mathrm{~s}^{-1}$, and the velocity with respect to the dark matter component becomes supersonic with Mach numbers of $\mathcal{M} \approx 5$ (Tseliakhovich and Hirata, 2010). In the subsequent cosmic evolution, the relative streaming velocity decays linearly with $z$, and reaches $\sim 1 \mathrm{~km} \mathrm{~s}^{-1}$ at the onset of first star formation at $z \approx 30$. Numerical simulations with streaming velocities suggest a reduction of the baryon overdensity in low-mass halos, a delay of the onset of cooling, and a larger critical mass for collapse (Greif et al., 2011: Stacy et al. 2011: Maio et al. 2011b; Naoz et al., 2012, 2013: O'Leary and McQuinn, 2012; Latif et al., 2014a; Schauer et al., 2017b). This can have substantial impact on the resulting $21 \mathrm{~cm}$ emission (Fialkov et al., 2012; McQuinn and O'Leary 2012 Visbal et al., 2012, see also Section 4). Gas in halos which are located in regions of large streaming velocities will be more turbulent than in more quiescent systems, and so we expect more fragmentation and a bias towards smaller stellar masses (Clark et al. 2008). However, this process has not yet been modeled with sufficient resolution, and so a reliable prediction of the stellar mass spectrum in regions with large streaming velocities is still outstanding.

\subsection{Dark matter annihilation}

Despite its importance for cosmic evolution and structure formation, the true physical nature of dark matter is unknown and so the studies of the impact of dark matter annihilation on first star formation are still highly speculative. As baryons collapse in the centers of primordial halos, they may drag along dark matter particles in a processes termed 'adiabatic contraction' (Blumenthal et al., 1986). It can lead to a density increase of several orders of magnitude, and as the annihilation rate scales quadratically with the density the corresponding energy input and ionization rate may become large enough to influence the dynamics of the gas. Spolyar et al. (2008) and 
Freese et al. (2009) suggest that this process could potentially overcome the cooling provided by $\mathrm{H}_{2}$ and eventually halt further collapse. They call the resulting object 'dark star'. It has a size of few astronomical units and is powered by dark matter annihilation rather than by nuclear fusion. If dark matter particles also scatter weakly on baryons this could lead to a structure that is stable for a long time (Freese et al., 2008; Iocco, 2008. Iocco et al., 2008, Yoon et al., 2008; Hirano et al., 2011).

There are several concerns that have been raised in this context. First, it is not clear whether collapse stalls once the energy input from dark matter annihilation becomes comparable to the cooling rate, because the larger heating rate may catalyze further $\mathrm{H}_{2}$ formation and lead to a correspondingly larger cooling rate (Ripamonti et al., 2010). Second, the underlying assumption of perfect alignment between dark matter cusp and gas collapse is most likely violated in realistic star formation conditions. For example, Stacy et al. (2012, 2014) find that non-axisymmetric perturbations lead to a separation between dark matter cusp and collapsing gas and causes the annihilation energy input to be too small for dark star formation. Similarly, Smith et al. (2012) see no evidence for the build-up of dark stars in their simulations. However, they find that dark matter annihilation has the potential to modify the dynamics of the accretion disk and reduce the level of disk fragmentation. As before, more work is required before we are able to draw firm conclusions.

\section{Supermassive stars}

Motivated by recent discoveries of supermassive black holes (SMBHs) exceeding $10^{9} \mathrm{M}_{\odot}$ at high redshifts $z \gtrsim 6$, the formation of supermassive stars (SMSs) in the early universe has drawn great attention for recent years. A possible formation pathway of the SMSs is the direct collapse (DC) model (e.g., Bromm and Loeb, 2003), where the SMSs gain the huge mass via very rapid mass accretion, typically $0.1-10 \mathrm{M}_{\odot} \mathrm{yr}^{-1}$ expected in atomic cooling primordial haloes.

Although the evolution and final fates of the SMSs had been investigated in the literature for past decades (e.g., Hoyle and Fowler, 1963, Osaki, 1966: Unno, 1971; Fricke, 1973; Fuller et al., 1986), the actual formation process had been ignored in such previous studies ('monolithic' models); a SMS in the stable nuclear burning phase was assumed initially, and then the subsequent evolution was followed. However, considering the formation process is critically important. For instance, the stellar radiative feedback against the accretion flow is a potential obstacle in forming massive stars. Even in the normal primordial star formation, where the typical stellar mass is thought to be $\sim 100 \mathrm{M}_{\odot}$, the stellar UV feedback may be strong enough to halt the mass accretion onto the stars (e.g., McKee and Tan, 2008, Hosokawa et al. 2011b: Sugimura et al. 2020). In the DC model, where the stellar mass is supposed to become much higher, the more tremendous UV radiative feedback may prevent the SMS formation early on. Solving the evolution of accreting SMSs from their birth stage is necessary to evaluate the strength of the UV feedback. In the same vein, the earlier evolution 
with the rapid mass accretion also affects the final fate of the SMS, i.e., at which point it collapses into a $\mathrm{BH}$. This determines how massive SMBH seeds are finally provided in the DC scenario.

\subsection{Numerical Modeling of Accreting Stars}

Numerical modeling of accreting stars has been developed in some different contexts, including the binary evolution (e.g., Kippenhahn and MeyerHofmeister, 1977; Neo et al. 1977), present-day star formation (e.g., Stahler et al., 1980, Stahler, 1988; Palla and Stahler, 1991; Behrend and Maeder, 2001) Hosokawa and Omukai 2009b Haemmerlé et al. 2016), and primordial star formation (e.g., Stahler et al., 1986; Omukai and Palla, 2003). In parallel with the emergence of the DC model, the similar method has been applied for the evolution of SMSs with extremely high accretion rates exceeding $10^{-2} \mathrm{M}_{\odot} \mathrm{yr}^{-1}$.

Regardless of such different contexts, the above studies basically solve the same governing equations to construct stellar models,

$$
\begin{gathered}
\left(\frac{\partial r}{\partial M}\right)_{t}=\frac{1}{4 \pi \rho r^{2}}, \\
\left(\frac{\partial P}{\partial M}\right)_{t}=-\frac{G M}{4 \pi r^{4}}, \\
\left(\frac{\partial L}{\partial M}\right)_{t}=\epsilon-T\left(\frac{\partial s}{\partial t}\right)_{M} \\
\left(\frac{\partial s}{\partial M}\right)_{t}=\frac{G M}{4 \pi r^{4}}\left(\frac{\partial s}{\partial p}\right)_{T}\left(\frac{L}{L_{s}}-1\right) C,
\end{gathered}
$$

where $M$ is the mass coordinate, $\epsilon$ the energy production rate via nuclear fusion, $s$ the specific entropy, $L_{s}$ the radiative luminosity with adiabatic temperature gradient, $C$ the coefficient determined by the mixing-length theory when $L>L_{s}(C=1$ otherwise), and the remaining quantities have ordinary meanings. Effects of the mass accretion are incorporated in the term $T(\partial s / \partial t)_{M}$ in Eq.(4), which is approximately discretized as

$$
\left(\frac{\partial s}{\partial t}\right)_{M}=\left(\frac{\partial s}{\partial t}\right)_{m}+\dot{M}_{*}\left(\frac{\partial s}{\partial m}\right)_{t},
$$

where $m$ is the inverse mass coordinate measured from the surface, $m \equiv$ $M_{*}-M$. An evolutionary calculation begins with an initial model with small mass, and advances with updating models by adding the accreting gas mass $\dot{M}_{*} \Delta t$, where $\Delta t$ is the time step. 


\subsubsection{Accretion-induced "Inflation" of Stars}

[Fig. 2 about here.]

Figure 2 summarizes the results by Hosokawa et al. (2012), who have numerically followed the evolution of protostars accreting at various constant rates including high rates exceeding $10^{-2} \mathrm{M}_{\odot} \mathrm{yr}^{-1}$. The black curve in panel (a) represents the case with $\dot{M}_{*}=10^{-3} \mathrm{M}_{\odot} \mathrm{yr}^{-1}$, a relatively low accretion rate typical for the normal primordial star formation. A striking feature for this case is the stellar contraction occurring for $6 \mathrm{M}_{\odot} \lesssim M_{*} \lesssim 30 \mathrm{M}_{\odot}$. This is the so-called Kelvin-Helmholtz $(\mathrm{KH})$ contraction stage, during which the star loses its energy by radiating away. The stellar effective temperature and emissivity of ionizing photons substantially rises during this, and the UV feedback against the accretion flows begins to operate. The presence of the KH contraction thus marks the onset the stellar UV feedback. Since the central temperature gradually rises, the hydrogen burning starts just after the stellar mass exceeds $30 \mathrm{M}_{\odot}$. After this point, the stellar radius turns to rise following the mass-radius relation for zero-age main-sequence (ZAMS) stars.

By contrast, the above evolution drastically changes with the more rapid accretion. Panel (a) shows that the $\mathrm{KH}$ contraction stage only spans the narrower mass range for such cases. The contraction turns to the expansion with the accretion rates $6 \times 10^{-3} \mathrm{M}_{\odot} \mathrm{yr}^{-1}$ and $0.03 \mathrm{M}_{\odot} \mathrm{yr}^{-1}$. With the highest rate $0.06 \mathrm{M}_{\odot} \mathrm{yr}^{-1}$, the contraction never occurs and the stellar radius monotonically increases as the star accretes the gas. The stellar radius finally reaches $\simeq 7000 \mathrm{R}_{\odot}$ (or nearly $\left.30 \mathrm{AU}\right)$ when the stellar mass is $\sim 1000 \mathrm{M}_{\odot}$. Panel (b) summarizes the evolution with the even higher accretion rates, $0.06 \mathrm{M}_{\odot} \mathrm{yr}^{-1} \leq \dot{M}_{*} \leq 1 \mathrm{M}_{\odot} \mathrm{yr}^{-1}$, showing further intriguing features, i.e., the curves corresponding to the different accretion rates represent the same mass-radius relation for $M_{*} \gtrsim 300 \mathrm{M}_{\odot}$. The radius at $M_{*} \simeq 1000 \mathrm{M}_{\odot}$ is consequently $\simeq 30 \mathrm{AU}$ for all the cases.

The above behavior is well interpreted with the following analytic argument. Let us begin with the general expression of the stellar luminosity

$$
L_{*}=4 \pi R_{*}^{2} \sigma T_{\mathrm{eff}}^{4},
$$

where $\sigma$ is the Stefan-Boltzmann constant. As for very massive stars, the luminosity is very close to the Eddington value,

$$
L_{*} \simeq L_{\mathrm{Edd}} \propto M_{*} .
$$

In the current cases with the high accretion rates, a stellar surface layer inflates because it always traps a part of the heat released from the contracting interior. The effective temperature accordingly decreases to several $\times 1000 \mathrm{~K}$, where $\mathrm{H}^{-}$bound-free absorption mostly contributes the opacity. Since the $\mathrm{H}^{-}$opacity has a very strong temperature-dependence, the effective temperature is almost fixed at a constant value. Substituting equation (8) and $T_{\text {eff }} \simeq 5000 \mathrm{~K}$ into equation (7), we derive the mass-radius relation

$$
R_{*} \simeq 2.6 \times 10^{3} R_{\odot}\left(\frac{M_{*}}{100 M_{\odot}}\right)^{1 / 2}
$$


which is indicated by the thin green lines in Figure 2. The above derivation includes no dependence on the accretion rate, which explains why the different lines in Figure 2 eventually converge into the same mass-radius relation.

\subsubsection{Birth of Accreting SMSs}

[Fig. 3 about here.]

Hosokawa et al. (2013) have further followed the later evolution until the stellar mass reaches $10^{4} \sim 10^{5} \mathrm{M}_{\odot}$. Figure 3 illustrates the evolution of the stellar interior structure with the constant accretion rate $1 \mathrm{M}_{\odot} \mathrm{yr}^{-1}$. We see that the monotonic increase of the stellar radius continues after the stellar mass exceeds $1000 \mathrm{M}_{\odot}$. The analytic formula given by equation $(9)$ still provides a good fit for the evolution of the stellar radius, though it only slightly overestimates the radius for $M_{*} \gtrsim 3 \times 10^{4} \mathrm{M}_{\odot}$. The similar evolution has been reported by the different authors (e.g., Umeda et al. 2016: Haemmerlé et al., 2018b).

Figure 3 shows that a surface layer which only has a small fraction of the total mass significantly inflates to cover a large part of the stellar radial extent. The star has a bloated envelope and compact core like red giants. Most of the stellar mass is contained in the central core, which gradually contracts as the star accretes the gas. The central temperature rises during this, as in the ordinary $\mathrm{KH}$ contraction. As a result, the nuclear burning starts just after the total stellar mass exceeds $\simeq 4000 \mathrm{M}_{\odot}$. The central convective core gradually extends after that, owing to the heat input caused by the nuclear fusion. The outer convective layer remains bloated as long as the rapid mass accretion continues, so that the stellar radius is much larger than the ZAMS stars with the same masses.

As described above, SMSs under the very rapid mass accretion greatly inflate. Their effective temperatures are only $\simeq 5000 \mathrm{~K}$, and the UV emissivity is much smaller than that of non-accreting stars. The resulting UV feedback is thus too weak to disturb the accretion flow toward the stars, so that the rapid growth of the stellar mass should continue as far as the high accretion rates exceeding $\sim 10^{-2} \mathrm{M}_{\odot} \mathrm{yr}^{-1}$ are maintained. In the standard DC model, SMSs exceeding $10^{5} \mathrm{M}_{\odot}$ may appear in less 0.1 Myr since the birth of the initial embryonic protostar, and they provides massive SMBH seeds once the gravitational collapse occurs via the general relativistic instability (Sect. 2.1.4).

\subsubsection{Accretion at $\dot{M}>10 \mathrm{M}_{\odot} \mathrm{yr}^{-1}$}

Beside the usual DC scenario based on atomic cooling haloes, an alternatice formation route for massive $\mathrm{BH}$ seeds has been proposed, relying on the merger of massive, gas-rich galaxies (Mayer et al., 2010, 2015; Mayer and Bonoli, 2019), as we describe in more details in Sect. 3. In this scenario, inflows as high as $10^{5} \mathrm{M}_{\odot} \mathrm{yr}^{-1}$ are found on subparsec scales.

The evolution of SMSs under accretion at $\dot{M}>10 \mathrm{M}_{\odot} \mathrm{yr}^{-1}$, up to $1000 \mathrm{M}_{\odot} \mathrm{yr}^{-1}$, has been simulated in Haemmerlé et al. (2019). The main 
qualitative difference compared to models at lower rates is that for such rates the accretion timescale $M / \dot{M}$ becomes similar to or shorter than the dynamical time of the star. Thus, above a limit in the accretion rate, the evolution proceeds hydrodynamically. This limit is an increasing function of the stellar mass $M$, since the perturbation to equilibrium induced by a given amount of mass added at the surface becomes relatively weaker as the stellar mass grows.

[Fig. 4 about here.]

The curve of $\dot{M}(M)$ is shown on Fig. 5. Rates of $\gtrsim 100 \mathrm{M}_{\odot} \mathrm{yr}^{-1}$ become consistent with equilibrium only when the accretor is supermassive $\left(M \gtrsim 10^{4} \mathrm{M}_{\odot}\right)$. If the conditions for equilibrium are satisfied, the star evolves in a qualitatively similar way as for lower rates: the envelope is inflated and the evolutionary track follows the Hayashi limit upwards. Most of the star is radiative, and hydrogen burns in the centre, driving convection in a core that contains a few percent of the total stellar mass. For $\dot{M}=1000 \mathrm{M}_{\odot} \mathrm{yr}^{-1}$, the mass fraction of the convective core can be as low as $1 \%$ at the endpoint of the evolution.

If lower mass objects accrete at rates $\gtrsim 100 \mathrm{M}_{\odot} \mathrm{yr}^{-1}$, their evolution proceeds dynamically, since the pressure and temperature gradients required for equilibrium cannot build. The further evolution in the dynamical regime is not known. In particular, the possibility for dark collapse (Mayer and Bonoli, 2019) shall be addressed with hydrodynamical simulations.

\subsubsection{The final collapse of SMSs}

SMSs end their life through the general relativistic (GR) instability, which is a radial pulsation instability that reflects photon's contribution to gravity (Chandrasekhar, 1964). GR effects impact negligibly the properties of stable SMSs, but they are critical for their stability, since these stars are always close to the Eddington limit. For $M>10^{4} \mathrm{M}_{\odot}$, the ratio $\beta$ of the gas pressure to the total pressure is of the order of a few percent. The first adiabatic exponent can be developed as

$$
\Gamma_{1}=\left.\frac{\mathrm{d} \ln P}{\mathrm{~d} \ln \rho}\right|_{\mathrm{ad}}=\frac{4}{3}+\frac{\beta}{6}+\mathcal{O}\left(\beta^{2}\right) .
$$

Thus, $\Gamma_{1}$ exceeds its classical critical value $\Gamma_{\text {crit }}=4 / 3$ by typically a percent. In these conditions, the small corrections to $\Gamma_{\text {crit }}$ arising from GR effects can trigger the collapse. For polytropic models, the first order postNewtonian correction to $\Gamma_{\text {crit }}$ is

$$
\Gamma_{\text {crit }}=\frac{4}{3}+K \frac{r_{S}}{r}+\mathcal{O}\left(\frac{r_{S}^{2}}{r^{2}}\right) .
$$

where $r_{S}=2 G M_{r} / c^{2}$ is the local Schwarzschild radius and $K$ is a constant that takes a value of 1.12 for a polytrope $n=3$, i.e. for an isentropic star 
in the limit $\beta \rightarrow 0$. As one considers increasing masses, the gas correction to $\Gamma_{1}$ decreases while the GR correction to $\Gamma_{\text {crit }}$ increases, until

$$
\frac{\beta}{6}<K \frac{r_{S}}{r} \quad \Longrightarrow \quad \Gamma_{1}<\Gamma_{\text {crit }}
$$

and the star becomes GR unstable.

[Fig. 5 about here.]

The limit in mass above which accreting SMSs become GR unstable has been investigated in numerical simulations (Umeda et al., 2016; Woods et al. 2017, Haemmerlé et al., 2018b), and are shown on Fig. 5. Discrepancies appear between the various studies (see Woods et al. 2019 for a detailed discussion), but all the models indicate a limit of several $10^{5} \mathrm{M}_{\odot}$ for a range in accretion rate that covers two orders of magnitudes. Models accreting at $100-1000 \mathrm{M}_{\odot} \mathrm{yr}^{-1}$ suggest that this limit remains always below $10^{6} \mathrm{M}_{\odot}$ even for such extreme rates (Haemmerlé et al., 2019).

Hydrodynamical simulations of the final collapse of SMSs indicate that the bulk of the stellar mass at the onset of instability forms a black hole in a dynamical time (Saijo et al., 2002; Saijo, 2004, Liu et al., 2007; Shibata et al., 2016b; Sun et al. 2017, 2018; Li et al., 2018, Uchida et al., 2017). In spherical symmetry, the whole mass is swallowed by the black hole, but rotation allows for a few percent of the stellar mass to orbit outside the horizon as a torus. Such asymmetries could trigger detectable gravitational wave (GW) emission. In the presence of a magnetic field, relativistic jets are found to form, which could trigger ultra-long gamma-ray bursts (ULGRBs). Thus the final collapse of SMSs might allow for multi-messenger signatures of DC. Thermonuclear explosion could occur in the presence of metals (Fuller et al. 1986; Montero et al., 2012), or in a narrow mass-range around $55000 \mathrm{M}_{\odot}($ Chen et al. 2014).

\subsection{Rotation in SMSs}

Rotation is key in the evolution of SMSs, for positive and negative reasons. The negative reasons rely on the angular momentum problem, which is general to star formation: the contraction from interstellar to stellar densities require mechanisms to extract angular momentum, in order to avoid break-up by centrifugal forces (Spitzer, 1978; Bodenheimer, 1995). This angular momentum barrier is particularly strong in massive star formation, due to the importance of radiation pressure, the lack of magnetic fields, and the short evolutionary timescales (e.g. Lee and Yoon 2016 Takahashi and Omukai 2017, Haemmerlé et al. 2017). It constitutes also a strong bottleneck for the efficient mass growth of SMBHs once formed (Sugimura et al. 2018). The positive reasons rely on the possibility for the final collapse of SMSs to trigger GWs and ULGRBs (Sect. 2.1.4). Rotation is required in both cases, in order to break spherical symmetry and to allow for the formation of collimated jets. 
The effect of rotation on SMSs has been studied in details in the context of monolithic models, i.e. neglecting accretion (Fowler, 1966; BisnovatyiKogan et al., 1967; Appenzeller and Kippenhahn, 1971; Baumgarte and Shapiro, 1999b a; New and Shapiro, 2001; Shibata et al., 2016a; Butler et al., 2018, Dennison et al., 2019). In general, these studies assume the star is rotating as a solid body, with a surface velocity corresponding to the Keplerian value. The assumption of solid rotation relies on convection, which dominates in monolithic models. The idea of Keplerian rotation follows from the argument of angular momentum conservation during the formation process. As a consequence of Keplerian rotation, the star is expected to loose mass at the equator as it further contracts, and this configuration is called the mass-shedding limit (Baumgarte and Shapiro, 1999b). The most striking result of these studies is the possibility for stars of $10^{8}-10^{9} \mathrm{M}_{\odot}$ to be stabilised by rotation against GR (Fowler, 1966, Bisnovatyi-Kogan et al. 1967). Moreover, the rotational properties of monolithic SMSs at collapse are found to be universal, in the sense that their spin parameter is

$$
a=\frac{c J}{G M^{2}}=0.97,
$$

according to numerical computations (Baumgarte and Shapiro, 1999a). This parameter is key for the outcome of the collapse, since a value $<1$ allows for the whole mass to form a black hole without angular momentum loss.

Monolithic models have been used as initial conditions to simulate the final collapse of SMSs (Saijo et al., 2002, Saijo, 2004; Liu et al., 2007; Shibata et al., 2016b, Sun et al., 2017, 2018, Li et al., 2018; Uchida et al., 2017). A black hole is found to form, containing $90-95 \%$ of the stellar mass, and a typical spin parameter $a \sim 0.7$. The remaining gas is maintained outside the horizon by the centrifugal force and takes the geometry of a torus, before relativistic jets are launched. Interestingly, relaxing the constraint of solid rotation allows for more angular momentum to be contained in the star, which results in more massive torus and more energetic jets (New and Shapiro, 2001; Sun et al., 2018).

When accretion at $\gtrsim 0.1 \mathrm{M}_{\odot} \mathrm{yr}^{-1}$ is accounted for, the rotational properties of SMSs are at the opposite extreme of those of monolithic models (Haemmerlé et al. 2018a, Haemmerlé and Meynet, 2019). First, since accretion stabilises the star against convection, solid-body rotation is not ensured, and the highly non-homologous contraction of the stellar interior leads to strong differential rotation, with a core rotating $10^{4}-10^{5}$ times faster than the surface in terms of frequency. Second, building a supermassive hydrostatic object by accretion requires its surface to rotate always at less than 10-20\% of the Keplerian velocity (Fig. 5). Thus, accreting SMSs must be slow rotators.

[Fig. 6 about here.]

This result is a consequence of the $\Omega \Gamma$-limit (Maeder and Meynet, 2000 ), i.e. the fact that the break-up velocity of massive stars is lowered by the effect of their strong radiation pressure, compared to Keplerian 
velocity. Break-up is reached when any contribution from gas pressure would destroy the star. For low-mass stars, this condition translates into the equality between gravity and the centrifugal force, which corresponds to the Keplerian limit:

$$
\frac{G M}{R_{\text {eq,crit }}^{2}}=\frac{\mathrm{v}_{\text {crit }, 1}^{2}}{R_{\text {eq,crit }}} \quad \Longleftrightarrow \quad \mathrm{v}_{\text {crit }, 1}=\sqrt{\frac{G M}{R_{\text {eq,crit }}}}=\sqrt{\frac{2}{3} \frac{G M}{R}}=\mathrm{v}_{\mathrm{Kep}},
$$

where $R_{\text {eq,crit }}$ is the equatorial radius at break-up, which according to the Roche model is 1.5 times the polar radius $R$, nearly unchanged by rotation. The Roche model is always accurate for SMSs, thanks to the large density contrasts in their interiors. For massive stars, one has to account for the effect of radiation pressure in the mechanical equilibrium, and the condition for break-up becomes

$$
\frac{G M}{R_{\text {eq,crit }}^{2}}=\frac{\mathrm{v}_{\text {crit }, 2}^{2}}{R_{\text {eq,crit }}}-\frac{1}{\rho} \frac{\mathrm{d} P_{\text {rad }}}{\mathrm{d} r},
$$

where $P_{\text {rad }}$ is the radiation pressure. If we neglect the deformation of the star and use the equation of radiative transfer $F=-\frac{c}{\kappa \rho} \frac{\mathrm{d} P_{\mathrm{rad}}}{\mathrm{d} r}$, this condition gives a break-up velocity

$$
\mathrm{v}_{\mathrm{crit}, 2}=\mathrm{v}_{\mathrm{crit}, 1} \sqrt{1-\frac{\kappa L}{4 \pi c G M}}=\mathrm{v}_{\mathrm{Kep}} \sqrt{1-\Gamma},
$$

where $\Gamma$ is the Eddington factor. This expression shows that in the limit $\Gamma \rightarrow 1$ (Eddington limit), the corrected critical velocity goes to 0, i.e. stars that are nearly Eddington must be slow rotators. When accounting for the anisotropy of the flux, in the limit $\Gamma \rightarrow 1$, the expression becomes

$$
\mathrm{v}_{\text {crit }, 2}=\frac{3}{2} \mathrm{v}_{\text {crit }, 1} \sqrt{1-\Gamma}
$$

Interestingly, for $\Gamma \lesssim 0.6$, one has $\mathrm{v}_{\text {crit, } 2}>\mathrm{v}_{\text {crit, } 1}$. This is a consequence of the von Zeipel theorem (von Zeipel, 1924), i.e. the reduction of the flux at the equator (where the centrifugal force is the strongest) due to the decrease of effective gravity. But for $\Gamma \gtrsim 0.6$, we see that $\mathrm{v}_{\text {crit }, 2}<\mathrm{v}_{\text {crit }, 1}=\mathrm{v}_{\mathrm{Kep}}$, i.e. the break-up velocity is lowered compared to the Keplerian limit.

Forming a supermassive hydrostatic object by accretion requires the constraint from the $\Omega \Gamma$-limit to be satisfied all along the accretion phase, for the in-falling gas to be incorporated by the star. As an extreme case of the $\Omega \Gamma$-limit, accreting SMSs cannot rotate faster than $10-20 \%$ of their Keplerian velocity (Fig. 5). It follows that their structure is not impacted by rotation. In addition, rotational mixing (shear diffusion and meridional circulation) remains negligible due to the short evolutionary timescales.

The constraint on the surface velocity translates into a constraint on the accreted angular momentum, which cannot exceed significantly a percent of the Keplerian angular momentum. The angular momentum barrier is thus particularly strong for SMSs. Two mechanisms might allow for efficient angular momentum loss in the accretion process: gravitational torques 
(Begelman et al., 2006, Wise et al., 2008, Hosokawa et al., 2016) and magnetic fields (Pandey et al., 2019; Haemmerlé and Meynet, 2019; Deng et al. 2019 , 2020). Supermassive accretion discs are highly gravitationally unstable, and departures from axial symmetry are pronounced. It results in strong gravitational torques that enhance accretion by removing angular momentum from the in-falling gas (Hosokawa et al., 2016). In addition, the turbulence driven by gravitational instability are found to generate strong magnetic fields, in a much more efficient way than magneto-rotational instability (Deng et al. 2019, 2020). These processes might be self-regulating, since any cut in accretion due to the centrifugal barrier will result in the accumulation of mass in the disc, which shall enhance fragmentation and turbulence. In this picture, the star will adjust on the fastest rotation velocity compatible with the $\Omega \Gamma$-limit, and the curve of Fig. 5 would give the expected rotation velocity of SMSs as a function of the mass, not only the upper limit. Magnetic fields might play a role at all the stages of the SMBH formation process. In the early phase of the collapse of the mini-halo, magnetic fields of $0.1 \mathrm{nG}$ are strong enough to avoid break-up (Pandey et al. 2019). Such fields are consistent with primordial fields whose flux was kept frozen during the formation of the halo. During the main accretion phase onto the star, magnetic coupling between the stellar surface and its winds could remove the $99 \%$ of the Keplerian angular momentum provided the magnetic field at the stellar surface is about $10 \mathrm{kG}$ (Haemmerlé and Meynet 2019). Such fields are too large to be driven by a convective dynamo, but they are consistent with primordial origins and correspond to the upper limit of detected magnetic fields on massive stars, which are suspected to be fossil fields (Townsend et al., 2005, Rivinius et al., 2010, Grunhut et al. 2012).

\section{Formation of massive black hole seeds}

Here we cover the different flavours of direct collapse scenarios that have been proposed in the literature. The formation of massive BH seeds from metal-free Population III stars is covered in Section 1. In this section we will also briefly discuss the growth, by accretion, of black hole seeds formed from Pop III stars and from direct collapse routes in light of the observational constraints currently posed by observations of bright QSOs at $z>5$.

[Fig. 7 about here.]

3.1 The rapid rise of high-z QSOs and the challenge for light black hole seed models

The existence of extremely bright QSOs up to $z>7$ (Fan et al., 2006, $\mathrm{Wu}$ et al. 2015, Bañados et al., 2018) is in tension with BH formation scenarios in which $\mathrm{BH}$ seeds start out light, of order a hundred solar masses as natural for the Pop III scenario (Sect. 1), and then grow gradually by Eddingtonlimited accretion. At $z>7$, assuming the standard LCDM cosmology, 
simply the lookback time is too short for a light $\mathrm{BH}$ seed to grow to $10^{9} \mathrm{M}_{\odot}$, the $\mathrm{BH}$ mass inferred by assuming that the source radiates at the Eddington limit (note that if accretion occurs at a fraction of Eddington, as suggested by other constraints for lower z QSOs, the timescale problem is further exacerbated). We briefly recall here the arguments that support the need for a massive $\mathrm{BH}$ seed in order to explain the rapid emergence of the bright high-z quasars. The time $t\left(M_{B H}\right)$ required for a black hole of initial mass $m_{\text {seed }}$ to reach a mass $M_{B H}$, assuming Eddington-limited and continous accretion, is given by:

$$
t\left(M_{B H}\right)=\frac{t_{E d d}}{f_{\text {edd }}} \frac{\epsilon_{r}}{(1-\eta)} \ln \left(\frac{M_{B H}}{m_{\text {seed }}}\right)
$$

where $t_{E d d} \equiv c \sigma_{T} /\left(4 \pi G \mu m_{p}\right)$ is the Eddinton time $(\sim 0.45$ Gyr for a pure hydrogen gas, with $\mu=1), f_{\text {edd }}$ expresses at which fraction of the Eddington limit the black hole is accreting, and $\epsilon_{r}$ and $\eta$ are, respectively, the radiative efficiency and accretion efficiency ${ }^{1}$. Assuming a radiativelyefficient accretion event, we assume $\epsilon_{r}=\eta=0.1$, a typical average value for Shakura-Sunyaev thin disks (Shakura and Sunyaev, 1973). Adopting a more realistic molecular weight per electron for a plasma at zero metallicity with cosmic abundance of hydrogen $(\mathrm{X}=0.75)$ and helium $(\mathrm{Y}=0.25)$, $\mu=1 /(1-Y / 2)=1.14$, the Eddington time lowers to $t_{E d d} \sim 0.39$ Gyr. Note that the precise value of the metallicity is marginal in this calculation, as metallicity has only a minor effect on the value of the molecular weight (e.g., for a gas at solar metallicity, $\mu=1.18$ instead of the value $\mu=1.14$ assumed here). The mass of the supermassive black holes powering the most luminous high-z quasars is typically of the order of $M_{B H}=10^{9} M_{\odot}$, so that this is the value we assume for the target mass. Note that this is a conservative choice as there is at least one known high-z QSO with black hole mass of $\sim 10^{10} M_{\odot}$, see Wu et al. $(2015)$. Further assuming that the seed black hole is able to accrete continuously at the Eddington limit $\left(f_{\text {edd }}=1\right)$, the timescale necessary to reach the target mass is $t_{\left(M_{B H}\right)}=0.4$ Gyr for a seed of $10^{5} M_{\odot}$, while is $t_{\left(M_{B H}\right)} \sim 0.7 \mathrm{Gyr}$ for a black holes starting from $10^{2} M_{\odot}$, the average value for light seeds produced by Pop III stars (see next paragraph and section 2). In this latter case the growth timescale is comparable to the age of the universe at $z=7$ (assuming the Planck cosmology of Ade et al. 2015).

There are two alternative solutions, which are being extensively studied since a few years. In the first one, the light $\mathrm{BH}$ seed is allowed to accrete above the Eddington limit, continuously or episodically. Pop III remnants can form black hole seeds with masses up to $\sim 1000 \mathrm{M}_{\odot}$ (see Sect. 1). The environments where Pop III BH seeds may form at $z \sim 15-30$ have been extensively studied with numerical simulations, which agree on the fact that such seeds grow too slow, at Sub-Eddington rates, as the gas in their host mini-halo is efficiently photoionized, and eventually expelled, owing

1 The accretion efficiency $\eta$ directly depends on the spin of the black hole, and reaches the maximum value of $\eta \sim 0.42$ for maximally rotating Kerr black holes. The radiative efficiency $\epsilon_{r}$ depends both on the type of accretion and on the accretion efficiency: for radiatively efficient accretion events, one can assume $\epsilon_{r}=\eta$. 
to radiative feedback from the massive stellar progenitor (eg., Johnson and Bromm 2007; Wise et al. 2008). In particular, primordial stars in the mass range $140-260 M_{\odot}$ explode as pair-instability supernovae (Heger et al. 2003), liberating enough energy to effectively empty the mini-halos of gas. Lower-mass Pop III stars would still damp energy, having a significant impact on the ambient medium if they form in clusters, as it is likely (Greif 2015), owing to the shallow potential wells of mini-halos. X-ray radiative feedback also suppresses further fueling of gas, leading effectively to accretion rates as small as $\sim 1 \%$ of the Eddington rate, and thus stifling the growth of the $\mathrm{BH}$ seed well below the expectations from the Eddingtonlimited accretion model considered above (Alvarez et al., 2009; Jeon et al. 2012).

Only later, when star formation has led to metal enrichment, increasing the cooling rate, and the original host halo has grown significantly in mass to allow atomic cooling via Ly- $\alpha$ radiation, accretion can in principle restart (see also the recent review by Inayoshi et al. 2019).

Super-Eddington accretion onto light seeds could then be achieved, in principle, in radiatively inefficient accretion disks or in accreting envelopes emitting highly anisotropically (see Mayer and Bonoli 2019 for a review, and Inayoshi et al. 2019; Takeo et al. 2018). Several studies, both at the level of radiation hydrodynamics and MHD simulations of various accretion flow configurations (e.g. Sadowski et al. 2013), and at the level of larger scale models at nuclear (proto) galactic scales with parametrized analytical accretion models, such as the SLIM disk model, have been developed in the last decades. Alternatively, the formation of a much heavier $\mathrm{BH}$ seed, with mass $>10^{4} M_{\odot}$ can be invoked, which could then grow in the required time to billion solar masses in an Eddington-limited regime. This second route is what we will discuss thoroughly in the remainder of this section, and goes by the name of direct collapse. Direct collapse is a general scenario rather than a specific model. There are indeed various models proposed to generate a massive $\mathrm{BH}$ seed without starting from a conventional stellar progenitor, as recently reviewed in detail by Woods et al. (2019). Two of the models examined in the literature are covered in the next subsection.

\subsection{Direct collapse scenarios for the origin of massive $\mathrm{BH}$ seeds}

In direct collapse scenarios the initial $\mathrm{BH}$ is not resulting from the collapse of an ordinary star. Note that, at high redshift $(z>20)$, for ordinary stars we mean Population III stars as described in the first section of this review. Population III stars would produce a light $\mathrm{BH}$ seed with mass in the range from a few tens to of order thousand solar masses, according to the mass distribution of such early population of metal-free stars. In direct collapse scenarios the $\mathrm{BH}$ seeds that arise have a mass of at least $10^{4} \mathrm{M}_{\odot}$, but can be even as large as $10^{8} \mathrm{M}_{\odot}$ in the most extreme models including rotation (Sect. 2.2) or through dark collapse (Mayer and Bonoli, 2019). The formation of an SMS is usually postulated for the precursor stage, which then collapses into a massive BH seed, by the GR radial instability, 
or because the SMS progenitor can never achieve a global hydrostatic equilibrium (see Sect. 2.1.3).

[Fig. 8 about here.]

\subsubsection{A crucial aspect: angular momentum transport in protogalaxies}

The first stage in the direct collapse scenarios involves gas skipping conventional star formation and reaching, instead, extremely high densities, collecting a gravitationally bound mass large enough to generate a supermassive precursor object. Note that we will assume this all happens in the galactic nucleus since QSOs are associated to galactic nuclei, but most of our considerations are not necessarily restricted to the nuclear region only. In order to generate a supermassive precursor, irrespective on whether or not the object will remain in a protostellar-like regime before collapsing, or will shine, even briefly, as an SMS, enormous quantities of interstellar gas have to be delivered to the galactic nucleus from galactic scales. This gas then has to become gravitationally bound and produce the precursor. These steps, necessary for the precursor stage, are a crucial aspect of direct collapse models and, while various propositions have been made, the jury is still on what is the actual mechanism that prevails, if any.

If there is general consensus on one aspect that is on the broad scenario for how angular momentum can be lost in protogalaxies. This is believed to happen primarily via gravitational torques in primordial gas-rich galaxies (e.g. Begelman et al. 2006, Lodato and Natarajan 2006, perhaps boosted by violent phenomena such as galaxy mergers (Mayer et al., 2010, 2015). In order for such torques to be effective at removing angular momentum from gas the galactic disks, and in particular their nuclear regions, need to be in a marginally self-gravitating state. This corresponds to a regime in which the Toomre Q parameter (Toomre, 1964), which measures the susceptibility of the disk to become unstable to its own self-gravity, must hover just above unity. In an entirely gaseous disk the Toomre parameter is defined as $Q=c_{s} \kappa / \pi G \Sigma$, where $c_{s}$ is the sound speed, $\kappa$ is the epicyclic frequency of gas motion, $\Sigma$ is the gas surface mass density, and $G$ is the gravitational constant. The definition is strictly local, although it is customary to use it also globally or expressing it using average properties in the disk. If $\mathrm{Q}$ drops below unity in most of the disk, gas will fragment copiously, producing substructure (clouds, and then stars from clouds possibly), thus interrupting the large scale global torquing action of spiral density waves by breaking their coherence. If, by heating or mass loss, it increases comfortably above unity $(Q>2)$ the disk will become gravitationally stable, and thus not capable anymore to transfer angular momentum and re-shuffle mass. If $Q$ remains low enough to allow self-gravity to excite non-axisymmetric structures, such as bars and spiral arms, efficient angular momentum transport can occur as gravitational torques by such structures behave as an effective viscous drag acting on the gas flow. Numerical simulations of protogalaxies have indeed shown that gas inflow rates are triggered by nonaxisymmetric instabilities, such as spiral arms (Latif et al., 2013a) and bars- 
in-bars instabilities (Shlosman, 2011, Choi et al., 2013). A clear example is shown in Figure 8.

Neglecting gas accretion, the stability of a gas-dominated protogalactic disk will be determined by the two conditions valid for any self-gravitating gas disk. Namely, its surface density must be low enough to maintain the Toomre Q above unity, and the cooling time has to be longer than the local orbital time (Gammie, 2001). For the latter condition, to so-called Gammie criterion), simulations using improved numerical resolution have demonstrated convergence as a function of resolution after a decade of discordant results (Deng et al. 2017 ). If the cooling time drops below the local orbital time the Toomre $\mathrm{Q}$ will decrease and eventually drop below unity, entering the fragmentation regime.

Models of direct collapse have thus focused on ways to avoid rapid cooling of the protogalactic disk in order to maintain sustained gas inflows as opposed to fragmentation. At sufficiently high redshift $(z>15-20)$ gas in typical dark matter halos was still metal-free (with the exception of rare high density peaks), and radiative cooling was occurring either via molecular lines, predominantly via roto-vibrational transitions of the $\mathrm{H}_{2}$ molecule, or, in sufficiently massive halos $\left(M_{v i r}>10^{7} M_{\odot}\right)$, via atomic hydrogen line cooling. If $\mathrm{H}_{2}$ cooling is suppressed, the protogalactic disk would not cool below a temperature of $\sim 10^{4} \mathrm{~K}$, thus evolving isothermally and remaining stable to fragmentation, as found in the semi-analytical model of Lodato and Natarajan (2006). Achieving suppression of molecular cooling has thus become the main objective in direct collapse models.

[Fig. 9 about here.]

\subsubsection{Routes to direct collapse: metal-free atomic cooling halos and mergers of most massive metal-enriched primordial galaxies}

In the last couple of years the community has made progress in studying both the conventional route to direct collapse, which involves gas inflows in metal-free atomic cooling halos (e.g. Latif et al.|2013a; Regan and Haehnelt 2009 Regan et al. 2017) and other scenarios, such as the merger-driven model (Mayer et al., 2010, Bonoli et al., 2014, Mayer and Bonoli, 2019), or models whereby colliding streams in proto-halos at very high-z (Hirano et al. 2017) or streaming velocities (Schauer et al., 2017a) play a role in providing favourable conditions.

The most conventional route to suppress $H_{2}$ cooling has been to invoke nearby sources of Lyman-Werner photons, which can efficiently dissociate $\mathrm{H}_{2}$. This could happen by invoking a proximity condition, as a sufficiently nearby star forming (proto)galaxy could be responsible for a high dissociating flux. The idea was proposed originally by Dijkstra et al. (2008), and explored first assuming a constant local dissociating Lyman-Werner background, to find out the required level in order to accomplish $H_{2}$ dissociation (e.g. Latif et al.|2013a), and, lately, computing self-consistently the spatially and temporally dependent dissociating flux from sources in galaxies forming within hydrodynamical cosmological simulations of the early Universe 
before the reionization epoch (Regan et al., 2017, 2019; Wise et al., 2019). A large body of theoretical and numerical work has led to the notion that the required Lyman-Werbner flux is roughly 1000 times higher than the reference intensity value $J_{21}=110^{-21} \mathrm{erg}^{-1} \mathrm{~cm}^{-2} \mathrm{~Hz}^{-1} \mathrm{sr}^{-1}$ (see review by Latif 2019), orders of magnitude above the expected mean background flux value (e.g. Ahn et al. 2009).

[Fig. 10 about here.]

Current state-of-the-art simulations, such as Renaissance (Wise et al. (2019), see Fig. 10), showed that the mechanism can be successful in very rare objects forming relatively late, at $z<20$, as it requires that, in addition to the proximity requirement, on one hand, sufficient metal-pollution to power gas cooling and intense star formation in the neighboring galaxy, and, on the other end, that the target system is still metal-free by that time. In the Renaissance cosmological volume, nearly $40 \mathrm{Mpc}$ on a side, out of which subvolumes encompassing high density regions were re-sampled at higher resolution, a few tens of candidate systems that remain starfree and metal-free by the onset of the atomic cooling halo phase were found (Regan et al., 2019). Most importantly, the Renaissance runs also showed that dynamical heating by mergers and accretion is contributing significantly to maintain a warm nearly isothermal core in the protogalaxy, hence relaxing the requirement on the critical Lyman-Werner flux. The few tens of atomic cooling halos that remain metal-free and star-free by the end of the simulation have indeed been exposed to a mean LymanWerner flux just comparable to $J_{21}$, yet the simulations are not conclusive as they lack the resolution to exclude fragmentation of the protogalactic cores into clusters of Pop III stars (Regan et al., 2019). In addition, even if fragmentation would not be an issue, the formation of a direct collapse $\mathrm{BH}$ seed would require sustained nuclear accretion rates at $>0.1 \mathrm{M}_{\odot} \mathrm{yr}^{-1}$ in the protogalactic nucleus to produce an SMS (see section 2), or even a quasi-star (Begelman, 2010), for timescales $>10^{6} \mathrm{yr}$ (namely at least longer than the expected lifetime of the SMS or quasi-star). In at least two of the highest resolution re-simulations of selected rare high density peaks in the Renaissance volume, such high inflow rate conditions seem indeed to be fulfilled towards the end of the simulation, almost $1 \mathrm{Myr}$ after the beginning of gas collapse in the halo (Wise et al., 2019). However, gas accumulation occurs on separate gravitationally bound clumps that, by the same time, achieve a mass only an order of magnitude higher than that of Pop III stars, between 1000 and $10^{4} \mathrm{M}_{\odot}$. With such the latter mass range for the precursors the resulting massive $\mathrm{BH}$ seed would still be too light to explain the most massive of the high-z QSOs $\left(M_{B H} \sim 10^{10} M_{\odot}\right)$ by Eddington limited accretion.

Mergers can also temporarily enhance gas inflow rates by removing angular momentum via strong tidal torques and gas shocking, these being considerably more intense than internal torques due to disk instabilities. The effect of dynamical heating brings us to the other proposed mechanism, namely heating by gas accretion and shocking induced mergers of much more massive and more mature galaxies at somewhat later epochs, $z \sim$ 
$8-10$. At this stage the first massive galaxies residing in halos with $M_{v i r} \sim 10^{12} \mathrm{M}_{\odot}$ do appear (Mayer et al., 2010). This mass scale is interesting since it is consistent with the virial mass expected for the hosts of the high-z QSOs given their clustering amplitude and low abundance (Volonteri et al., 2008).

In this second scenario, since it occurs much later, gas is already metalenriched, yet simulations have shown that cooling is still inefficient as compressional heating and shocks in equal mass mergers are sufficient to counteract cooling by maintaining the temperature of the pc-scale disky core emerging in the merger remnant at $5000-8000 \mathrm{~K}$, allowing only sporadic fragmentation at its outskirts (Mayer et al. 2015, see Figure 10). Most importantly, in this case radial gas inflows, and thus gas accretion rates onto the core, occur at the phenomenal rate in the range $1000-$ $10^{4} \mathrm{M}_{\odot} \mathrm{yr}^{-1}$ for nearly $10^{5} \mathrm{yr}$ after the merger (Figure 11), allowing the formation of an ultra-compact supermassive rotating gas core that thus meets naturally the conditions to become an SMS (see Sect. 2.1.3). This allows the central core to reach a mass of $\sim 10^{9} M_{\odot}$ in less than $10^{5}$ yr. The central core is fast rotating and gravitationally bound, and with such a high mass it can form, in principle, a supermassive BH seed which would have no problem in reaching the mass of the brightest QSOs in less than hundred million years even with accretion sustained at a fraction of Eddington (Bonoli et al., 2014, Mayer and Bonoli, 2019).

The reason why inflow rates are so much higher in the merger-driven collapse is partially because major mergers produce the strongest tidal torques and shocks, as they give rise to the largest displacement of mass, and partially because the halos involved in this scenario are, by construction, much more massive than atomic cooling halos. Indeed, to zeroth order, neglecting residual angular momentum in the flow, the radial gas flow will displace mass with amplitude $\dot{M} \sim V_{v i r}^{3} / G \sim M_{v i r}$ (where $M_{v i r}$ and $V_{v i r}$ are the virial mass and virial velocity of the host halo, respectively), with $M_{v i r}$ increasing by more than a factor of 1000 between atomic cooling halos $\left(M_{v i r} \sim 10^{9-10} M_{\odot}\right)$ and high-sigma peaks at $z \sim 8-10$ possessing $M_{v i r} \sim 10^{12} M_{\odot}$ (see Bonoli et al. 2014: Mayer and Bonoli 2019 for details). New multi-scale merger simulations of high density peaks reaching parsec resolution with fully cosmological initial conditions (from the previously published MassiveBlack runs of Feng and Soria 2011) appear to confirm the existence of such prominent gas inflows (Capelo, Mayer et al., in preparation). With such extreme conditions it is also possible that the SMS stage is skipped, and rather a BH seed nearly as massive as the entire supermassive gas core could form either because it becomes compact enough to collapse due to the General Relativistic radial instability (Mayer and Bonoli, 2019), or because it can never find an hydrostatic equilibrium configuration (see Sect. 2.1.3), a route refereed to as dark collapse. In this latter case an ultra-massive $\mathrm{BH}$ seed would arise, as massive as nearly the entire precursor core of $\sim 10^{9} M_{\odot}$, since numerical relativity simulations of rotating polytropic SMS/clouds reaching a critical compactness show that the radial GR instability triggers a global collapse of about half of the total mass into a central black hole (e.g. Shibata and Shapiro 2002), or even into 
a binary system of two black holes if the massive precursor becomes bar unstable and fragments (Reisswig et al., 2013).

Observationally, both the conventional direct collapse scenario based on $\mathrm{H}_{2}$ dissociation in metal-free atomic cooling halos and the merger-driven scenario gives rise to a number of direct collapse $\mathrm{BHs}$ that, while low in absolute sense, it is still high enough to accomodate the abundance of high-z QSOs (Mayer and Bonoli, 2019, Latif, 2019). However, a sensible quantitative comparison with observations will have to take into account the detailed mechanism of $\mathrm{BH}$ seed formation from the precursor, eg whether an SMS, a quasi-star, or neither of the two, will form prior to collapse (see Woods et al. 2019), and the duty-cycle of the resulting AGN.

\section{Reionization after Planck and before JWST}

The transformation of cold neutral intergalactic hydrogen into a highly ionized warm plasma marks the end of the dark ages and the beginning of the age of galaxies. In popular cosmological scenarios, massive stars within early protogalaxies, aided perhaps by a population of accreting seed black holes, generated the UV and X-ray radiation that reheated and reionized the Universe. Observations of resonant absorption in the spectra of distant quasars show that hydrogen reionization was still ongoing at $z \sim 6$ and fully completed by redshift 5.5 (Fan et al., 2006, Becker et al. 2015). An early onset of reionization appears to be disfavored by the final, full-mission Planck CMB anisotropy analysis, which yields an integrated Thomson scattering optical depth of $\tau_{\text {es }}=0.054 \pm 0.007$ (Planck Collaboration et al. 2018). And while the evolution of the ionization and thermal state of the $z \gtrsim 6$ Universe is being constrained by a number of direct probes, from the damping wing absorption profiles in the spectra of quasars (e.g., Bañados et al. 2017), through the luminosity function (LF) and clustering properties of Ly $\alpha$ emitting galaxies (Ouchi et al., 2010, Schenker et al., 2014), to the flux power spectrum of the Ly $\alpha$ forest (Oñorbe et al., 2017), many pivotal aspects of this process remain highly uncertain.

[Fig. 11 about here.]

Over the last few years, HST deep optical and NIR imaging data have led to the discovery and study of some of the earliest-known galaxies at redshifts $z=7-10$, corresponding to a period when the Universe was

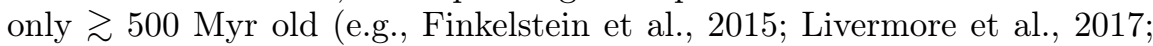
Oesch et al., 2017). The faint-end slope of the rest-frame UV LF of these high-redshift sources has been measured to be very steep $(\alpha \approx-2)$, such that the integrated UV luminosity density is contributed equally by each decade in galaxy luminosity, and therefore may depend on whatever physics regulates star formation in the faintest dwarfs (see, e.g., Bouwens et al. 2012, Atek et al., 2015; Ishigaki et al., 2017, and references therein). The importance of star-forming galaxies for reionization further depends on the Lyman-continuum (LyC) photon yield per unit UV luminosity and on the fraction of these photons that can escape into the IGM (Madau et al. 1999 
Robertson et al. 2010). Galaxy-dominated scenarios that satisfy many of the observational constraints can be constructed if one extrapolates the UV LF to sufficiently faint magnitudes $\left(M_{\mathrm{UV}} \sim-13\right)$ assuming a time- and luminosity-averaged escape fraction of $\sim 15 \%$ at $z \gtrsim 6$ (e.g., Haardt and Madau, 2012, Robertson et al., 2015, Bouwens et al., 2015, Madau, 2017). The presence of moderate- and low-luminosity AGNs at early times can change inferences on the role of galaxies in reionization (e.g., Madau and Haardt, 2015, Giallongo et al., 2019), and the differing effective temperature of the radiation between stars and AGN can impact the thermal history of the reionized IGM (D'Aloisio et al., 2017). At low metallicity, stellar populations in early galaxies may be characterized by an increased LyC photon production from binary stars compared to the present-day Universe (Stanway et al., 2016 ). The enhanced LyC photon yield may boost the equivalent width of nebular emission lines ([O III], $\mathrm{H} \alpha, \mathrm{H} \beta$ ) (e.g., Smit et al., 2014), which are redshifted into the rest-frame wavelength range relevant for the James Webb Space Telescope (JWST) NIRSpec instrument. The impact of nebular emission on the spectra of galaxies is also regulated by the escape fraction, and forthcoming measurements of the rest-frame UV continuum slope with the equivalent width of the $\mathrm{H} \beta$ emission line may allow the identification of galaxies with extreme LyC leakage up to redshift 9 (Zackrisson et al., 2017).

[Fig. 12 about here.]

Figures 12 and 13 depict some of the available observational probes of the epoch of reionization. These include observations of the Gunn-Peterson absorption troughs indicating that the Universe was mostly reionized by $z \sim 6$ (Fan et al., 2006, Becker et al., 2015), the evolution of the cosmic SFR density at high redshift (Oesch et al., 2017), and the evolution of the IGM mean temperature at $z \lesssim 5.5$ (Walther et al., 2019). In addition, the sensitivity of Ly $\alpha$ emission to intervening neutral gas is also being used to explain the dearth of Ly $\alpha$ emitters (LAEs) observed at $z>6.5$ (Ouchi et al. 2010, Schenker et al., 2014). Recent Planck CMB anisotropy data together with measurements of the kinetic Sunyaev-Zel'dovich effect by the Atacama Cosmology and the South Pole Telescopes constrain the redshift where the volume-averaged neutral fraction drops below 50\% to lie between $z=7.3$ and $10.5(2 \sigma)$, and yield an upper limit to the width of the reionization period of $\Delta z<2.8$ (Planck Collaboration et al. 2016b).

[Fig. 13 about here.]

\subsection{The reionization equation}

Cosmic reionization involves a complex interplay between the abundance, clustering, spectrum, and LyC radiation leakage of photoionizing sources, and the density, clumpiness, and temperature of intergalactic gas. Detailed simulations of reionization must be informed by insight from semi-empirical modeling and theoretical calculations that provide a proper accounting of 
the volume-averaged production and absorption of ionizing radiation in an expanding medium. Below, we give an example of how theory informs computation.

The general idea of a gradual reionization process driven by a steadily increasing UV photon production rate can be cast into a simple quantitative framework by integrating the "reionization equation" (Madau, 2017)

$$
\frac{d\left\langle x_{\mathrm{HII}}\right\rangle}{d t}=\frac{\left\langle\dot{n}_{\gamma}\right\rangle}{\left\langle n_{\mathrm{H}}\right\rangle\left(1+\left\langle\kappa_{\nu_{L}}^{\mathrm{LLS}}\right\rangle /\left\langle\kappa_{\nu_{L}}^{\mathrm{IGM}}\right\rangle\right)}-\frac{\left\langle x_{\mathrm{HII}}\right\rangle}{\bar{t}_{\mathrm{rec}}} .
$$

for the time evolution of the volume-averaged hydrogen ionized fraction $x_{\mathrm{HII}}$. Here, $\left\langle\dot{n}_{\gamma}\right\rangle$ is the emission rate into the IGM of ionizing photons per unit proper volume, $\left\langle n_{\mathrm{H}}\right\rangle$ is the cosmological mean proper density of hydrogen, $\left\langle\kappa^{\mathrm{LLS}}\right\rangle^{-1}$ and $\left\langle\kappa^{\mathrm{IGM}}\right\rangle^{-1}$ are the photon proper mean free path at the hydrogen Lyman edge caused by the Lyman-limit systems (LLSs) and the diffuse IGM, respectively, and $\bar{t}_{\text {rec }}$ is an effective recombination timescale in a clumpy IGM ${ }^{2}$ The above equation assumes a photon mean free path that is much smaller than the horizon size (so that the mean specific intensity relaxes to the source function), and an early IGM that is organized in three main phases - a uniform ionized medium, a uniform neutral medium, and the LLSs (Madau, 2017).

[Fig. 14 about here.]

It is this ODE that statistically describes the transition from a neutral universe to a fully ionized one, turning reionization into a photon-counting exercise in which the growth rate of $\mathrm{H}$ II regions is equal to the rate at which ionizing photons are produced minus the rate at which they are consumed by radiative recombinations in the IGM. Before reionization, at $z>6$, UV photons carve out cosmological $\mathrm{H}$ II regions in the uniform component that expand in an otherwise largely neutral phase. At $z<6$, however, the diffuse IGM is observed to be highly ionized, with only a small residual amount of neutral hydrogen set by the balance between radiative recombinations and photoionizations from a nearly uniform UV radiation background, and provides negligibly small $\mathrm{H}$ i photoelectric absorption. The continuum opacity is instead dominated by the optically thick (to ionizing radiation) LLSs, high density regions that trace non-linear and collapsed structures, occupy a small portion of the volume, and are able to keep a significant fraction of their hydrogen in neutral form (e.g. Miralda-Escudé et al., 2000, Gnedin and Fan, 2006, Furlanetto and Mesinger, 2009; Haardt and Madau, 2012). The integration of Equation (19) therefore provides a link between the pre-overlap and post-overlap phases of the reionization process.

\footnotetext{
2 Photon losses by LLSs are also due to radiative recombinations. In analytical models it is standard practice, however, to include the effect of LLSs as a reduction in the source term through the finite mean free path of ionizing radiation. Three different quantities - the escape fraction, the gas clumping factor, and the mean free path - are then used to describe what are essentially radiative recombinations in the ISM, the IGM, and the LLSs. Numerical simulations of cosmic reionization show that such a separation into distinct regimes may indeed be reasonable (Kaurov and Gnedin, 2015).
} 
Figure 14 shows the reionization history obtained by numerically integrating Equation (19) assuming a constant emission rate of ionizing photons per hydrogen atom, $\left\langle\dot{n}_{\gamma}\right\rangle /\left\langle n_{\mathrm{H}}\right\rangle=3$ phot $\mathrm{Gyr}^{-1}$. The theoretical curve is consistent with a number of observational constraints on the ionization state of the $z>5$ universe, from the redshift-dependent prevalence of LAEs in narrow band surveys at $z=7-8$ (Schenker et al. 2014), to the damping wing absorption profiles measured in the spectra of $z=6.3$ (Schroeder et al. 2013) and $z=7.1$ quasars (Greig et al., 2017). While the pre-overlap stages extend over a considerable range of redshift, the phase of overlap, indicated by the sudden drop in the neutral gas fraction at $z \simeq 6$, is clearly defined even in the presence of the LLSs: $\left\langle x_{\mathrm{HI}}\right\rangle$ decreases by more than 2 orders of magnitude over a fraction of the then Hubble time. Such a dramatic transition marks the epoch when the photon mean free path becomes determined by the LLSs rather than by the typical size of $\mathrm{H}$ II regions. Note how current state-of-the-art radiation-hydrodynamic simulations of reionization produce a poor fit to the observations of the Gunn-Peterson optical depth in the spectra of Sloan Digital Sky Survey (SDSS) quasars at $5<z<61$ (Fan et al. 2006), as well as to estimates of the ionized fraction at $z=5$ and 6 based on a combination of hydrodynamical simulations with measurements of the Ly $\alpha$ forest opacity (Bolton and Haehnelt, 2007). The disagreement is an artifact of current reionization simulations underestimating the amount of UV absorption in the post-reionization Universe, as LLSs remain effectively unresolved.

\section{Concluding remarks}

We have reviewed our current understanding of early structure formation, from first stars to the first black holes emerging at high redshift, and have addressed the impact of early star forming galaxies and early accreting massive black holes on the reionization of the Universe. We have highlighted the importance of radiative feedback in determining various key aspects of first objects, such as the masses of first stars and the growth rate of the black holes arising from them. Radiative feedback, in particular dissociation of molecular hydrogen by the Lyman-Werner radiation produced by early star forming galaxies, plays a key role also in models for massive black hole seed formation $\left(M_{B H}>10^{4} M_{\odot}\right.$ by direct collapse in metal-free atomic cooling halos. Likewise, thermodynamical feedback from accretion and mergers could provide the required heating to avoid fragmentation in the most rapidly growing atomic cooling halos. Alternative models for direct collapse, such as from major mergers of the first metal-enriched massive galaxies at $z \sim 8-10$, actually rely exclusively on thermodynamical feedback from shocks and compressional heating to stifle widespread fragmentation, and can give rise to much larger black hole seeds, of order a billion solar mass. In direct collapse models, a paramount role is played by the physics of their likely precursors, accreting supermassive stars (SMS). We have thus covered in detail the latest developments in modeling SMSs, including rotation and the different regimes arising with accretion rates varying by orders of magnitude, according to either the atomic cooling halo scenario 
and the galaxy merger-driven scenario. In doing so, we have reported on the possibility that collapse into a massive black hole seed, at the highest accretion rates, occurs without prior formation of an SMS, the so-called dark collapse route. The reionization of hydrogen during the first billion years of cosmic history is the last major phase transition of baryonic matter in the Universe and involves the early production, propagation, and absorption of $\mathrm{LyC}$ radiation. The full reionization of helium is believed to have occurred at a later time, when hard UV-emitting bright quasars and other AGN became sufficiently abundant. While it is generally agreed that star-forming galaxies and the first accreting seed black holes in their nuclei are the best candidates for providing the required ionizing power, there are still many uncertainties on the relative contributions of these sources as a function of epoch. The resolution of such uncertanties feels overdue and may soon be achieved by the large wavelength coverage, unique sensitivity, and spectroscopic and imaging capabilities of the JWST together with ongoing and future experiments aimed at measuring the redshifted $21-\mathrm{cm}$ signal from neutral hydrogen during the epoch of first light.

\section{References}

T. Abel, G.L. Bryan, M.L. Norman, The Formation of the First Star in the Universe. Science 295, 93-98 (2002)

T. Abel, J.H. Wise, G.L. Bryan, The H II Region of a Primordial Star. ApJ 659, 87-90 (2007)

P.A.R. Ade, Y. Akiba, A.E. Anthony, et al., a Measurement of the Cosmic Microwave Background B-Mode Polarization with Polarbear. Publication of Korean Astronomical Society 30(2), 625-628 (2015)

K. Ahn, P.R. Shapiro, I.T. Iliev, et al., The Inhomogeneous Background Of H2-Dissociating Radiation During Cosmic Reionization. ApJ 695, 1430-1445 (2009)

M.A. Alvarez, V. Bromm, P.R. Shapiro, The H II Region of the First Star. ApJ 639, 621-632 (2006)

M.A. Alvarez, J.H. Wise, T. Abel, Accretion onto the First Stellar-Mass Black Holes. ApJ 701, 133-137 (2009)

I. Appenzeller, R. Kippenhahn, General Relativistic Secular Instability of Supermassive Stars. A\&A 11, 70 (1971)

H. Atek, J. Richard, M. Jauzac, et al., Are Ultra-faint Galaxies at z $=6$ 8 Responsible for Cosmic Reionization? Combined Constraints from the Hubble Frontier Fields Clusters and Parallels. ApJ 814, 69 (2015)

E. Bañados, B.P. Venemans, C. Mazzucchelli, et al., An 800 million solar mass black hole in a significantly neutral universe at redshift 7.5. ArXiv e-prints (2017)

E. Bañados, B.P. Venemans, C. Mazzucchelli, et al., An 800-million-solarmass black hole in a significantly neutral Universe at a redshift of 7.5. Nature 553, 473-476 (2018)

S.A. Balbus, J.F. Hawley, Instability, turbulence, and enhanced transport in accretion disks. Reviews of Modern Physics 70, 1-53 (1998) 
R. Banerjee, K. Jedamzik, Are Cluster Magnetic Fields Primordial? Physical Review Letters 91(25), 251301 (2003)

R. Barnett, S.J. Warren, G.D. Becker, et al., Observations of the Lyman series forest towards the redshift 7.1 quasar ULAS J1120+0641. A\&A 601, 16 (2017)

T.W. Baumgarte, S.L. Shapiro, Evolution of Rotating Supermassive Stars to the Onset of Collapse. ApJ 526, 941-952 (1999a)

T.W. Baumgarte, S.L. Shapiro, Luminosity versus Rotation in a Supermassive Star. ApJ 526, 937-940 (1999b)

G.D. Becker, J.S. Bolton, P. Madau, et al., Evidence of patchy hydrogen reionization from an extreme Ly $\alpha$ trough below redshift six. MNRAS 447, 3402-3419 (2015)

M.C. Begelman, Evolution of supermassive stars as a pathway to black hole formation. MNRAS 402, 673-681 (2010)

M.C. Begelman, M. Volonteri, M.J. Rees, Formation of supermassive black holes by direct collapse in pre-galactic haloes. MNRAS 370, 289-298 (2006)

R. Behrend, A. Maeder, Formation of massive stars by growing accretion rate. A\&A 373, 190-198 (2001)

L. Biermann, Über den Ursprung der Magnetfelder auf Sternen und im interstellaren Raum (miteinem Anhang von A. Schlüter). Zeitschrift Naturforschung Teil A 5, 65 (1950)

J. Binney, S. Tremaine, Galactic dynamics (Princeton University Press, ???, 1987)

G.S. Bisnovatyi-Kogan, Y.B. Zel'dovich, I.D. Novikov, Evolution of Supermassive Stars Stabilized by Large-Scale Motions. SvA 11, 419 (1967)

G.R. Blumenthal, S.M. Faber, R. Flores, et al., Contraction of dark matter galactic halos due to baryonic infall. ApJ 301, 27-34 (1986)

P. Bodenheimer, Angular Momentum Evolution of Young Stars and Disks. ARA\&A 33, 199-238 (1995)

J.S. Bolton, M.G. Haehnelt, The observed ionization rate of the intergalactic medium and the ionizing emissivity at $\mathrm{z}>=5$ : evidence for a photon-starved and extended epoch of reionization. MNRAS 382, 325$341(2007)$

S. Bonoli, L. Mayer, S. Callegari, Massive black hole seeds born via direct gas collapse in galaxy mergers: their properties, statistics and environment. MNRAS 437, 1576-1592 (2014)

R.J. Bouwens, G.D. Illingworth, P.A. Oesch, et al., Lower-luminosity Galaxies Could Reionize the Universe: Very Steep Faint-end Slopes to the UV Luminosity Functions at $\mathrm{z}>=5-8$ from the HUDF09 WFC3/IR Observations. ApJ 752, 5 (2012)

R.J. Bouwens, G.D. Illingworth, P.A. Oesch, et al., Reionization After Planck: The Derived Growth of the Cosmic Ionizing Emissivity Now Matches the Growth of the Galaxy UV Luminosity Density. ApJ 811, $140(2015)$

S. Bovino, D.R.G. Schleicher, J. Schober, Turbulent magnetic field amplification from the smallest to the largest magnetic Prandtl numbers. New Journal of Physics 15(1), 013055 (2013) 
V. Bromm, R.B. Larson, The First Stars. ARA\&A 42, 79-118 (2004)

V. Bromm, A. Loeb, Formation of the First Supermassive Black Holes. ApJ 596, 34-46 (2003)

V. Bromm, P.S. Coppi, R.B. Larson, The Formation of the First Stars. I. The Primordial Star-forming Cloud. ApJ 564, 23-51 (2002)

S.P. Butler, A.R. Lima, T.W. Baumgarte, et al., Maximally rotating supermassive stars at the onset of collapse: the perturbative effects of gas pressure, magnetic fields, dark matter, and dark energy. MNRAS 477, 3694-3710 (2018)

S. Chandrasekhar, The Dynamical Instability of Gaseous Masses Approaching the Schwarzschild Limit in General Relativity. ApJ 140, 417 (1964)

K.-J. Chen, A. Heger, S. Woosley, et al., The General Relativistic Instability Supernova of a Supermassive Population III Star. ApJ 790, 162 (2014)

J.-H. Choi, I. Shlosman, M.C. Begelman, Supermassive Black Hole Formation at High Redshifts via Direct Collapse: Physical Processes in the Early Stage. ApJ 774, 149 (2013)

P.C. Clark, S.C.O. Glover, R.S. Klessen, The First Stellar Cluster. ApJ 672, 757-764 (2008)

P.C. Clark, S.C.O. Glover, R.S. Klessen, et al., Gravitational Fragmentation in Turbulent Primordial Gas and the Initial Mass Function of Population III Stars. ApJ 727, 110 (2011a)

P.C. Clark, S.C.O. Glover, R.J. Smith, et al., The Formation and Fragmentation of Disks Around Primordial Protostars. Science 331, 1040 (2011b)

B. Commerçon, P. Hennebelle, T. Henning, Collapse of Massive Magnetized Dense Cores Using Radiation Magnetohydrodynamics: Early Fragmentation Inhibition. ApJ 742, 9 (2011)

A. D'Aloisio, P.R. Upton Sanderbeck, M. McQuinn, et al., On the contribution of active galactic nuclei to the high-redshift metagalactic ionizing background. MNRAS 468, 4691-4701 (2017)

H. Deng, L. Mayer, H. Latter, Global simulations of self-gravitating magnetized protoplanetary disks. arXiv e-prints, 2001-08693 (2020)

H. Deng, L. Mayer, F. Meru, Convergence of the Critical Cooling Rate for Protoplanetary Disk Fragmentation Achieved: The Key Role of Numerical Dissipation of Angular Momentum. ApJ 847(1), 43 (2017)

H. Deng, L. Mayer, H. Latter, et al., Local Simulations of MRI turbulence with Meshless Methods. ApJS 241(2), 26 (2019)

K.A. Dennison, T.W. Baumgarte, S.L. Shapiro, Maximally Rotating Supermassive Stars at the Onset of Collapse: Effects of Gas Pressure. arXiv e-prints, 1906-04190 (2019)

M. Dijkstra, Z. Haiman, A. Mesinger, et al., Fluctuations in the highredshift Lyman-Werner background: close halo pairs as the origin of supermassive black holes. MNRAS 391, 1961-1972 (2008)

G. Dopcke, S.C.O. Glover, P.C. Clark, et al., On the Initial Mass Function of Low-metallicity Stars: The Importance of Dust Cooling. ApJ 766, 103 (2013)

A. Doussot, H. Trac, R. Cen, SCORCH. II. Radiation-Hydrodynamic simulations of reionization with varying radiation escape fractions. ArXiv e-prints (2017) 
X. Fan, M.A. Strauss, R.H. Becker, et al., Constraining the Evolution of the Ionizing Background and the Epoch of Reionization with z $\sim 6$ Quasars. II. A Sample of 19 Quasars. AJ 132, 117-136 (2006)

C. Federrath, S. Sur, D.R.G. Schleicher, et al., A New Jeans Resolution Criterion for (M)HD Simulations of Self-gravitating Gas: Application to Magnetic Field Amplification by Gravity-driven Turbulence. ApJ 731, $62(2011)$

C. Federrath, J. Schober, S. Bovino, et al., The Turbulent Dynamo in Highly Compressible Supersonic Plasmas. ApJ 797, 19 (2014)

H. Feng, R. Soria, Ultraluminous X-ray sources in the Chandra and XMMNewton era. Nature 55, 166-183 (2011)

A. Fialkov, R. Barkana, D. Tseliakhovich, et al., Impact of the relative motion between the dark matter and baryons on the first stars: semianalytical modelling. MNRAS 424, 1335-1345 (2012)

S.L. Finkelstein, R.E. Ryan Jr., C. Papovich, et al., The Evolution of the Galaxy Rest-frame Ultraviolet Luminosity Function over the First Two Billion Years. ApJ 810, 71 (2015)

W.A. Fowler, The Stability of Supermassive Stars. ApJ 144, 180 (1966)

K. Freese, P. Bodenheimer, D. Spolyar, et al., Stellar Structure of Dark Stars: A First Phase of Stellar Evolution Resulting from Dark Matter Annihilation. ApJ 685, 101 (2008)

K. Freese, P. Bodenheimer, P. Gondolo, et al., Dark stars: a new study of the first stars in the Universe. New Journal of Physics 11(10), 105014 (2009)

K.J. Fricke, Dynamical Phases of Supermassive Stars. ApJ 183, 941-958 (1973)

G.M. Fuller, S.E. Woosley, T.A. Weaver, The evolution of radiationdominated stars. I - Nonrotating supermassive stars. ApJ 307, 675-686 (1986)

S.R. Furlanetto, A. Mesinger, The ionizing background at the end of reionization. MNRAS 394, 1667-1673 (2009)

S. Gallerani, A. Ferrara, X. Fan, et al., Glimpsing through the high-redshift neutral hydrogen fog. MNRAS 386, 359-369 (2008)

C.F. Gammie, Nonlinear Outcome of Gravitational Instability in Cooling, Gaseous Disks. ApJ 553, 174-183 (2001)

E. Giallongo, A. Grazian, F. Fiore, et al., Space Densities and Emissivities of Active Galactic Nuclei at z \&gt; 4. ApJ 884(1), 19 (2019)

P. Girichidis, C. Federrath, R. Banerjee, et al., Importance of the initial conditions for star formation - I. Cloud evolution and morphology. MNRAS 413, 2741-2759 (2011)

P. Girichidis, C. Federrath, R. Banerjee, et al., Importance of the initial conditions for star formation - II. Fragmentation-induced starvation and accretion shielding. MNRAS 420, 613-626 (2012a)

P. Girichidis, C. Federrath, R. Allison, et al., Importance of the initial conditions for star formation - III. Statistical properties of embedded protostellar clusters. MNRAS 420, 3264-3280 (2012b)

S. Glover, The Formation Of The First Stars In The Universe. Space Sci. Rev. 117, 445-508 (2005) 
S. Glover, The First Stars, in The First Galaxies, ed. by T. Wiklind, B. Mobasher, V. Bromm Astrophysics and Space Science Library, vol. 396, 2013, p. 103

N.Y. Gnedin, X. Fan, Cosmic Reionization Redux. ApJ 648, 1-6 (2006)

D. Grasso, H.R. Rubinstein, Magnetic fields in the early Universe. Phys. Report 348, 163-266 (2001)

T.H. Greif, The numerical frontier of the high-redshift Universe. Computational Astrophysics and Cosmology 2, 3 (2015)

T.H. Greif, J.L. Johnson, R.S. Klessen, et al., The first galaxies: assembly, cooling and the onset of turbulence. MNRAS 387, 1021-1036 (2008)

T.H. Greif, S.D.M. White, R.S. Klessen, et al., The Delay of Population III Star Formation by Supersonic Streaming Velocities. ApJ 736, 147 (2011)

T.H. Greif, V. Bromm, P.C. Clark, et al., Formation and evolution of primordial protostellar systems. MNRAS 424, 399-415 (2012)

B. Greig, A. Mesinger, Z. Haiman, et al., Are we witnessing the epoch of reionisation at $\mathrm{z}=7.1$ from the spectrum of J1120 +0641 ? MNRAS 466, 4239-4249 (2017)

J.H. Grunhut, T. Rivinius, G.A. Wade, et al., HR 5907: Discovery of the most rapidly rotating magnetic early B-type star by the MiMeS Collaboration. MNRAS 419, 1610-1627 (2012)

F. Haardt, P. Madau, Radiative Transfer in a Clumpy Universe. IV. New Synthesis Models of the Cosmic UV/X-Ray Background. ApJ 746, 125 (2012)

L. Haemmerlé, G. Meynet, Magnetic braking of supermassive stars through winds. A\&A 623, 7 (2019)

L. Haemmerlé, P. Eggenberger, G. Meynet, et al., Massive star formation by accretion. I. Disc accretion. A\&A 585, 65 (2016)

L. Haemmerlé, P. Eggenberger, G. Meynet, et al., Massive star formation by accretion. II. Rotation: how to circumvent the angular momentum barrier? A\&A 602, 17 (2017)

L. Haemmerlé, T.E. Woods, R.S. Klessen, et al., On the Rotation of Supermassive Stars. ApJ 853, 3 (2018a)

L. Haemmerlé, T.E. Woods, R.S. Klessen, et al., The evolution of supermassive Population III stars. MNRAS 474, 2757-2773 (2018b)

L. Haemmerlé, G. Meynet, L. Mayer, et al., Maximally accreting supermassive stars: a fundamental limit imposed by hydrostatic equilibrium. A\&A 632, 2 (2019)

A. Heger, C.L. Fryer, S.E. Woosley, et al., How Massive Single Stars End Their Life. ApJ 591, 288-300 (2003)

S. Hirano, H. Umeda, N. Yoshida, Evolution of Primordial Stars Powered by Dark Matter Annihilation up to the Main-sequence Stage. ApJ 736, $58(2011)$

S. Hirano, T. Hosokawa, N. Yoshida, et al., One Hundred First Stars: Protostellar Evolution and the Final Masses. ApJ 781, 60 (2014)

S. Hirano, T. Hosokawa, N. Yoshida, et al., Primordial star formation under the influence of far ultraviolet radiation: 1540 cosmological haloes and the stellar mass distribution. MNRAS 448, 568-587 (2015) 
S. Hirano, T. Hosokawa, N. Yoshida, et al., Supersonic gas streams enhance the formation of massive black holes in the early universe. Science $\mathbf{3 5 7}$, 1375-1378 (2017)

T. Hosokawa, K. Omukai, Evolution of Massive Protostars with High Accretion Rates. ApJ 691, 823-846 (2009a)

T. Hosokawa, K. Omukai, Evolution of Massive Protostars with High Accretion Rates. ApJ 691, 823-846 (2009b)

T. Hosokawa, K. Omukai, H.W. Yorke, Rapidly Accreting Supergiant Protostars: Embryos of Supermassive Black Holes? ApJ 756, 93 (2012)

T. Hosokawa, H.W. Yorke, K. Omukai, Evolution of Massive Protostars Via Disk Accretion. ApJ 721, 478-492 (2010)

T. Hosokawa, K. Omukai, N. Yoshida, et al., Protostellar Feedback Halts the Growth of the First Stars in the Universe. Science 334, 1250 (2011a)

T. Hosokawa, K. Omukai, N. Yoshida, et al., Protostellar Feedback Halts the Growth of the First Stars in the Universe. Science 334, 1250 (2011b)

T. Hosokawa, N. Yoshida, K. Omukai, et al., Protostellar Feedback and Final Mass of the Second-generation Primordial Stars. ApJ 760, 37 (2012)

T. Hosokawa, H.W. Yorke, K. Inayoshi, et al., Formation of Primordial Supermassive Stars by Rapid Mass Accretion. ApJ 778, 178 (2013)

T. Hosokawa, S. Hirano, R. Kuiper, et al., Formation of Massive Primordial Stars: Intermittent UV Feedback with Episodic Mass Accretion. ApJ 824, 119 (2016)

F. Hoyle, W.A. Fowler, On the nature of strong radio sources. MNRAS 125, 169 (1963)

K. Inayoshi, E. Visbal, Z. Haiman, The Assembly of the First Massive Black Holes. arXiv e-prints, 1911-05791 (2019)

F. Iocco, Dark Matter Capture and Annihilation on the First Stars: Preliminary Estimates. ApJ 677, 1 (2008)

F. Iocco, A. Bressan, E. Ripamonti, et al., Dark matter annihilation effects on the first stars. MNRAS 390, 1655-1669 (2008)

M. Ishigaki, R. Kawamata, M. Ouchi, et al., Full-Data Results of Hubble Frontier Fields: UV Luminosity Functions at $z \sim 6-10$ and a Consistent Picture of Cosmic Reionization. ArXiv e-prints (2017)

M. Jeon, A.H. Pawlik, T.H. Greif, et al., The First Galaxies: Assembly with Black Hole Feedback. ApJ 754, 34 (2012)

M. Jeon, A.H. Pawlik, V. Bromm, et al., Radiative feedback from high-mass $\mathrm{X}$-ray binaries on the formation of the first galaxies and early reionization. MNRAS 440, 3778-3796 (2014)

J.L. Johnson, V. Bromm, The aftermath of the first stars: massive black holes. MNRAS 374, 1557-1568 (2007)

A.A. Kaurov, N.Y. Gnedin, Cosmic Reionization on Computers. III. The Clumping Factor. ApJ 810, 154 (2015)

A.P. Kazantsev, Enhancement of a Magnetic Field by a Conducting Fluid. Soviet Journal of Experimental and Theoretical Physics 26, 1031 (1968)

E. Keto, The Formation of Massive Stars: Accretion, Disks, and the Development of Hypercompact H II Regions. ApJ 666, 976-981 (2007)

R. Kippenhahn, E. Meyer-Hofmeister, On the radii of accreting main 
sequence stars. A\&A 54, 539-542 (1977)

T. Kitayama, N. Yoshida, H. Susa, et al., The Structure and Evolution of Early Cosmological H II Regions. ApJ 613, 631-645 (2004)

R.S. Klessen, S.C.O. Glover, Physical Processes in the Interstellar Medium. Star Formation in Galaxy Evolution: Connecting Numerical Models to Reality, Saas-Fee Advanced Course, Volume 43. ISBN 978-3-662-478899. Springer-Verlag Berlin Heidelberg, 2016, p. 85 43, 85 (2016)

K.M. Kratter, C.D. Matzner, Fragmentation of massive protostellar discs. MNRAS 373, 1563-1576 (2006)

M.R. Krumholz, R.I. Klein, C.F. McKee, et al., The Formation of Massive Star Systems by Accretion. Science 323, 754 (2009)

R. Kuiper, H. Klahr, H. Beuther, et al., Circumventing the Radiation Pressure Barrier in the Formation of Massive Stars via Disk Accretion. ApJ 722, 1556-1576 (2010)

R. Kuiper, H. Klahr, H. Beuther, et al., Three-dimensional Simulation of Massive Star Formation in the Disk Accretion Scenario. ApJ 732, 20 (2011)

R.B. Larson, Numerical calculations of the dynamics of collapsing protostar. MNRAS 145, 271 (1969)

M.A. Latif, J.C. Niemeyer, D.R.G. Schleicher, Impact of baryonic streaming velocities on the formation of supermassive black holes via direct collapse. MNRAS 440, 2969-2975 (2014a)

M.A. Latif, D.R.G. Schleicher, W. Schmidt, Magnetic fields during the formation of supermassive black holes. MNRAS 440, 1551-1561 (2014b)

M.A. Latif, D.R.G. Schleicher, W. Schmidt, et al., High-resolution studies of massive primordial haloes. MNRAS 430, 588-598 (2013a)

M.A. Latif, D.R.G. Schleicher, W. Schmidt, et al., The small-scale dynamo and the amplification of magnetic fields in massive primordial haloes. MNRAS 432, 668-678 (2013b)

M.A. Latif, Black hole formation via gas-dynamical processes, ed. by M. Latif, D. Schleicher 2019, pp. 99-113

M. Lazar, R. Schlickeiser, R. Wielebinski, et al., Cosmological Effects of Weibel-Type Instabilities. ApJ 693, 1133-1141 (2009)

H. Lee, S.-C. Yoon, On the Role of the $\Omega \Gamma$ Limit in the Formation of Population III Massive Stars. ApJ 820, 135 (2016)

J.-T. Li, G.M. Fuller, C.T. Kishimoto, Neutrino burst-generated gravitational radiation from collapsing supermassive stars. Phys. Rev. D 98(2), $023002(2018)$

Y.T. Liu, S.L. Shapiro, B.C. Stephens, Magnetorotational collapse of very massive stars to black holes in full general relativity. Phys. Rev. D 76(8), 084017 (2007)

R.C. Livermore, S.L. Finkelstein, J.M. Lotz, Directly Observing the Galaxies Likely Responsible for Reionization. ApJ 835, 113 (2017)

G. Lodato, P. Natarajan, Supermassive black hole formation during the assembly of pre-galactic discs. MNRAS 371, 1813-1823 (2006)

M.-M. Mac Low, R.S. Klessen, Control of star formation by supersonic turbulence. Reviews of Modern Physics 76, 125-194 (2004)

M.N. Machida, K. Doi, The formation of Population III stars in gas 
accretion stage: effects of magnetic fields. MNRAS 435, 3283-3305 (2013)

M.N. Machida, T. Matsumoto, S.-i. Inutsuka, Magnetohydrodynamics of Population III Star Formation. ApJ 685, 690-704 (2008)

M.N. Machida, K. Omukai, T. Matsumoto, et al., The First Jets in the Universe: Protostellar Jets from the First Stars. ApJ 647, 1-4 (2006)

P. Madau, Cosmic Reionization after Planck and before JWST: An Analytic Approach. ApJ 851, 50 (2017)

P. Madau, F. Haardt, Cosmic Reionization after Planck: Could Quasars Do It All? ApJ 813, 8 (2015)

P. Madau, F. Haardt, M.J. Rees, Radiative Transfer in a Clumpy Universe. III. The Nature of Cosmological Ionizing Sources. ApJ 514, 648-659 (1999)

A. Maeder, Physics, Formation and Evolution of Rotating Stars (Springer, ???, 2009)

A. Maeder, G. Meynet, Stellar evolution with rotation. VI. The Eddington and Omega -limits, the rotational mass loss for OB and LBV stars. A\&A 361, 159-166 (2000)

U. Maio, L.V.E. Koopmans, B. Ciardi, The impact of primordial supersonic flows on early structure formation, reionization and the lowest-mass dwarf galaxies. MNRAS 412, 40-44 (2011a)

U. Maio, S. Khochfar, J.L. Johnson, et al., The interplay between chemical and mechanical feedback from the first generation of stars. MNRAS 414, 1145-1157 (2011b)

L. Mayer, S. Bonoli, The route to massive black hole formation via mergerdriven direct collapse: a review. Reports on Progress in Physics 82(1), 016901 (2019)

L. Mayer, S. Kazantzidis, A. Escala, et al., Direct formation of supermassive black holes via multi-scale gas inflows in galaxy mergers. Nature $\mathbf{4 6 6}$, 1082-1084 (2010)

L. Mayer, D. Fiacconi, S. Bonoli, et al., Direct Formation of Supermassive Black Holes in Metal enriched Gas at the Heart of High-redshift Galaxy Mergers. ApJ 810, 51 (2015)

I.D. McGreer, A. Mesinger, V. D'Odorico, Model-independent evidence in favour of an end to reionization by z ApJ 6. MNRAS 447, 499-505 (2015)

C.F. McKee, E.C. Ostriker, Theory of Star Formation. ARA\&A 45, 565687 (2007)

C.F. McKee, J.C. Tan, The Formation of the First Stars. II. Radiative Feedback Processes and Implications for the Initial Mass Function. ApJ 681, 771-797 (2008)

M. McQuinn, R.M. O'Leary, The Impact of the Supersonic Baryon-Dark Matter Velocity Difference on the $\mathrm{z}^{\sim} 2021 \mathrm{~cm}$ Background. ApJ 760, 3 (2012)

M.V. Medvedev, L.O. Silva, M. Fiore, et al., Generation of Magnetic Fields in Cosmological Shocks. Journal of Korean Astronomical Society 37, 533$541(2004)$

J. Miralda-Escudé, M. Haehnelt, M.J. Rees, Reionization of the Inhomoge- 
neous Universe. ApJ 530, 1-16 (2000)

P.J. Montero, H.-T. Janka, E. Müller, Relativistic Collapse and Explosion of Rotating Supermassive Stars with Thermonuclear Effects. ApJ 749, $37(2012)$

S. Naoz, N. Yoshida, N.Y. Gnedin, Simulations of Early Baryonic Structure Formation with Stream Velocity. I. Halo Abundance. ApJ 747, 128 (2012)

S. Naoz, N. Yoshida, N.Y. Gnedin, Simulations of Early Baryonic Structure Formation with Stream Velocity. II. The Gas Fraction. ApJ 763, 27 (2013)

S. Neo, S. Miyaji, K. Nomoto, et al., Effect of Rapid Mass Accretion onto the Main-Sequence Stars. PASJ 29, 249-262 (1977)

K.C.B. New, S.L. Shapiro, Evolution of Differentially Rotating Supermassive Stars to the Onset of Bar Instability. ApJ 548, 439-446 (2001)

J. Oñorbe, J.F. Hennawi, Z. Lukić, et al., Constraining Reionization with the z 5-6 Ly-alpha Forest Power Spectrum: The Outlook after Planck. ApJ 847, 63 (2017)

P.A. Oesch, R.J. Bouwens, G.D. Illingworth, et al., The Dearth of z $\sim 10$ Galaxies in all HST Legacy Fields - The Rapid Evolution of the Galaxy Population in the First 500 Myr. ArXiv e-prints (2017)

R.M. O'Leary, M. McQuinn, The Formation of the First Cosmic Structures and the Physics of the $\mathrm{z}^{\sim} 20$ Universe. ApJ 760, 4 (2012)

K. Omukai, Primordial Star Formation under Far-Ultraviolet Radiation. ApJ 546, 635-651 (2001)

K. Omukai, F. Palla, Formation of the First Stars by Accretion. Apj 589, 677-687 (2003)

Y. Osaki, The Pulsation and Evolution of Super-Massive Stars. PASJ 18, $384(1966)$

B.W. O'Shea, M.L. Norman, Population III Star Formation in a $\Lambda \mathrm{CDM}$ Universe. I. The Effect of Formation Redshift and Environment on Protostellar Accretion Rate. ApJ 654, 66-92 (2007)

M. Ouchi, K. Shimasaku, H. Furusawa, et al., Statistics of 207 Ly $\alpha$ Emitters at a Redshift Near 7: Constraints on Reionization and Galaxy Formation Models. ApJ 723, 869-894 (2010)

F. Palla, S.W. Stahler, The evolution of intermediate-mass protostars. I Basic results. ApJ 375, 288-299 (1991)

K.L. Pandey, S.K. Sethi, B. Ratra, Cosmological magnetic braking and the formation of high-redshift, super-massive black holes. arXiv e-prints (2019)

M.V. Penston, Dynamics of self-gravitating gaseous spheres-III. Analytical results in the free-fall of isothermal cases. MNRAS 144, 425 (1969)

T. Peters, M.-M. Mac Low, R. Banerjee, et al., Understanding Spatial and Spectral Morphologies of Ultracompact H II Regions. ApJ 719, 831-843 (2010)

T. Peters, R. Banerjee, R.S. Klessen, et al., The Interplay of Magnetic Fields, Fragmentation, and Ionization Feedback in High-mass Star Formation. ApJ 729, 72 (2011)

T. Peters, D.R.G. Schleicher, R.J. Smith, et al., Low-metallicity star 
formation: relative impact of metals and magnetic fields. MNRAS 442, 3112-3126 (2014)

Planck Collaboration, P.A.R. Ade, N. Aghanim, et al., Planck 2015 results. XIII. Cosmological parameters. A\&A 594, 13 (2016a)

Planck Collaboration, R. Adam, N. Aghanim, et al., Planck intermediate results. XLVII. Planck constraints on reionization history. A\&A 596, 108 (2016b)

Planck Collaboration, N. Aghanim, Y. Akrami, et al., Planck 2018 results. VI. Cosmological parameters. ArXiv e-prints (2018)

D. Rahner, E.W. Pellegrini, S.C.O. Glover, et al., Winds and radiation in unison: a new semi-analytic feedback model for cloud dissolution. \mnras 470(4), 4453-4472 (2017)

D. Rahner, E.W. Pellegrini, S.C.O. Glover, et al., WARPFIELD 2.0: feedback-regulated minimum star formation efficiencies of giant molecular clouds. \mnras 483(2), 2547-2560 (2019)

J.A. Regan, M.G. Haehnelt, The formation of compact massive selfgravitating discs in metal-free haloes with virial temperatures of ${ }^{\sim} 13000$ 30000K. MNRAS 393, 858-871 (2009)

J.A. Regan, E. Visbal, J.H. Wise, et al., Rapid formation of massive black holes in close proximity to embryonic protogalaxies. Nature Astronomy 1, 0075 (2017)

J.A. Regan, T.P. Downes, M. Volonteri, et al., Super-Eddington accretion and feedback from the first massive seed black holes. MNRAS 486(3), 3892-3906 (2019)

C. Reisswig, C.D. Ott, E. Abdikamalov, et al., Formation and Coalescence of Cosmological Supermassive-Black-Hole Binaries in Supermassive-Star Collapse. Physical Review Letters 111(15), 151101 (2013)

E. Ripamonti, F. Iocco, A. Ferrara, et al., First star formation with dark matter annihilation. MNRAS 406, 2605-2615 (2010)

T. Rivinius, T. Szeifert, L. Barrera, et al., Magnetic field detection in the B2Vn star HR 7355. MNRAS 405, 46-50 (2010)

B.E. Robertson, R.S. Ellis, J.S. Dunlop, et al., Early star-forming galaxies and the reionization of the Universe. Nature 468, 49-55 (2010)

B.E. Robertson, R.S. Ellis, S.R. Furlanetto, et al., Cosmic Reionization and Early Star-forming Galaxies: A Joint Analysis of New Constraints from Planck and the Hubble Space Telescope. ApJ 802, 19 (2015)

A.L. Rosen, M.R. Krumholz, C.F. McKee, et al., An unstable truth: how massive stars get their mass. MNRAS 463, 2553-2573 (2016)

A. Sadowski, R. Narayan, R. Penna, et al., Energy, momentum and mass outflows and feedback from thick accretion discs around rotating black holes. MNRAS 436, 3856-3874 (2013)

M. Saijo, The Collapse of Differentially Rotating Supermassive Stars: Conformally Flat Simulations. ApJ 615, 866-879 (2004)

M. Saijo, T.W. Baumgarte, S.L. Shapiro, et al., Collapse of a Rotating Supermassive Star to a Supermassive Black Hole: Post-Newtonian Simulations. ApJ 569, 349-361 (2002)

D. Schaerer, On the properties of massive Population III stars and metalfree stellar populations. A\&A 382, 28-42 (2002) 
A.T.P. Schauer, B. Agarwal, S.C.O. Glover, et al., Lyman-Werner escape fractions from the first galaxies. MNRAS 467, 2288-2300 (2017a)

A.T.P. Schauer, J. Regan, S.C.O. Glover, et al., The formation of direct collapse black holes under the influence of streaming velocities. MNRAS 471, 4878-4884 (2017b)

A.T.P. Schauer, D.J. Whalen, S.C.O. Glover, et al., Lyman-Werner UV escape fractions from primordial haloes. MNRAS (2015)

A.A. Schekochihin, S.C. Cowley, G.W. Hammett, et al., A model of nonlinear evolution and saturation of the turbulent MHD dynamo. New Journal of Physics 4, 84 (2002)

A.A. Schekochihin, S.C. Cowley, S.F. Taylor, et al., Saturated State of the Nonlinear Small-Scale Dynamo. Physical Review Letters 92(8), 084504 (2004)

M.A. Schenker, R.S. Ellis, N.P. Konidaris, et al., Line-emitting Galaxies beyond a Redshift of 7: An Improved Method for Estimating the Evolving Neutrality of the Intergalactic Medium. ApJ 795, 20 (2014)

D.R.G. Schleicher, D. Galli, S.C.O. Glover, et al., The Influence of Magnetic Fields on the Thermodynamics of Primordial Star Formation. ApJ 703, 1096-1106 (2009)

D.R.G. Schleicher, R. Banerjee, S. Sur, et al., Small-scale dynamo action during the formation of the first stars and galaxies. I. The ideal MHD limit. A\&A 522, 115 (2010)

R. Schlickeiser, P.K. Shukla, Cosmological Magnetic Field Generation by the Weibel Instability. ApJ 599, 57-60 (2003)

J. Schober, D. Schleicher, C. Federrath, et al., Magnetic field amplification by small-scale dynamo action: Dependence on turbulence models and Reynolds and Prandtl numbers. Phys. Rev. E 85(2), 026303 (2012a)

J. Schober, D. Schleicher, C. Federrath, et al., The Small-scale Dynamo and Non-ideal Magnetohydrodynamics in Primordial Star Formation. ApJ 754, 99 (2012b)

J. Schober, D.R.G. Schleicher, C. Federrath, et al., Saturation of the turbulent dynamo. Phys. Rev. E 92(2), 023010 (2015)

J. Schroeder, A. Mesinger, Z. Haiman, Evidence of Gunn-Peterson damping wings in high-z quasar spectra: strengthening the case for incomplete reionization at z 6-7. MNRAS 428, 3058-3071 (2013)

N.I. Shakura, R.A. Sunyaev, Black holes in binary systems. Observational appearance. A\&A 24, 337-355 (1973)

M. Shibata, S.L. Shapiro, Collapse of a Rotating Supermassive Star to a Supermassive Black Hole: Fully Relativistic Simulations. ApJ 572, 39-43 (2002)

M. Shibata, H. Uchida, Y.-i. Sekiguchi, Stability of Rigidly Rotating Supermassive Stars against Gravitational Collapse. ApJ 818, 157 (2016a)

M. Shibata, Y. Sekiguchi, H. Uchida, et al., Gravitational waves from supermassive stars collapsing to a supermassive black hole. Phys. Rev. D 94(2), 021501 (2016b)

I. Shlosman, Galactic bars in cosmological context. Memorie della Societa Astronomica Italiana Supplementi 18, 175 (2011)

F.H. Shu, Self-similar collapse of isothermal spheres and star formation. 
ApJ 214, 488-497 (1977)

G. Sigl, A.V. Olinto, K. Jedamzik, Primordial magnetic fields from cosmological first order phase transitions. Phys. Rev. D 55, 4582-4590 (1997)

J. Silk, The cosmic microwave background radiation - Implications for galaxy formation, ed. by E.W. Kolb, M.S. Turner, D. Lindley, et al.(University of Chicago Press, 1986), pp. 143-158

R. Smit, R.J. Bouwens, I. Labbé, et al., Evidence for Ubiquitous Highequivalent-width Nebular Emission in $\mathrm{z}^{\sim} 7$ Galaxies: Toward a Clean Measurement of the Specific Star-formation Rate Using a Sample of Bright, Magnified Galaxies. ApJ 784, 58 (2014)

R.J. Smith, S.C.O. Glover, P.C. Clark, et al., The effects of accretion luminosity upon fragmentation in the early universe. MNRAS 414,3633$3644(2011)$

R.J. Smith, T. Hosokawa, K. Omukai, et al., Variable accretion rates and fluffy first stars. MNRAS 424, 457-463 (2012)

L. Spitzer, Physical processes in the interstellar medium (New York: WileyInter science, ???, 1978)

D. Spolyar, K. Freese, P. Gondolo, Dark Matter and the First Stars: A New Phase of Stellar Evolution. Physical Review Letters 100(5), 051101 (2008)

A. Stacy, V. Bromm, Constraining the statistics of Population III binaries. MNRAS 433, 1094-1107 (2013)

A. Stacy, V. Bromm, A.T. Lee, Building up the Population III initial mass function from cosmological initial conditions. MNRAS 462, 1307-1328 (2016)

A. Stacy, V. Bromm, A. Loeb, Effect of Streaming Motion of Baryons Relative to Dark Matter on the Formation of the First Stars. ApJ 730, 1 (2011)

A. Stacy, T.H. Greif, V. Bromm, The first stars: mass growth under protostellar feedback. MNRAS 422, 290-309 (2012)

A. Stacy, A.H. Pawlik, V. Bromm, et al., The mutual interaction between Population III stars and self-annihilating dark matter. MNRAS 441, 822-836 (2014)

S.W. Stahler, Deuterium and the stellar birthline. ApJ 332, 804-825 (1988)

S.W. Stahler, F. Palla, E.E. Salpeter, Primordial stellar evolution - The protostar phase. ApJ 302, 590-605 (1986)

S.W. Stahler, F.H. Shu, R.E. Taam, The evolution of protostars. I - Global formulation and results. ApJ 241, 637-654 (1980)

E.R. Stanway, J.J. Eldridge, G.D. Becker, Stellar population effects on the inferred photon density at reionization. MNRAS 456, 485-499 (2016)

K. Subramanian, Can the turbulent galactic dynamo generate large-scale magnetic fields? MNRAS 294, 718 (1998)

K. Sugimura, T. Hosokawa, H. Yajima, et al., Stunted accretion growth of black holes by combined effect of the flow angular momentum and radiation feedback. MNRAS 478(3), 3961-3975 (2018)

K. Sugimura, T. Matsumoto, T. Hosokawa, et al., The Birth of a Massive First-Star Binary. arXiv e-prints, 2002-00012 (2020)

L. Sun, M. Ruiz, S.L. Shapiro, Simulating the magnetorotational collapse 
of supermassive stars: Incorporating gas pressure perturbations and different rotation profiles. Phys. Rev. D 98(10), 103008 (2018)

L. Sun, V. Paschalidis, M. Ruiz, et al., Magnetorotational collapse of supermassive stars: Black hole formation, gravitational waves, and jets. Phys. Rev. D 96(4), 043006 (2017)

R.A. Sunyaev, Y.B. Zel'dovich, Small-Scale Fluctuations of Relic Radiation. Ap\&SS 7, 3-19 (1970)

S. Sur, D.R.G. Schleicher, R. Banerjee, et al., The Generation of Strong Magnetic Fields During the Formation of the First Stars. ApJ 721, 134138 (2010)

S. Sur, C. Federrath, D.R.G. Schleicher, et al., Magnetic field amplification during gravitational collapse - influence of turbulence, rotation and gravitational compression. MNRAS 423, 3148-3162 (2012)

H. Susa, The Mass of the First Stars. ApJ 773, 185 (2013)

H. Susa, K. Hasegawa, N. Tominaga, The Mass Spectrum of the First Stars. ApJ 792, 32 (2014)

S.Z. Takahashi, K. Omukai, Primordial protostars accreting beyond the $\Omega \Gamma$-limit: radiation effect around the star-disc boundary. MNRAS 472, $532-541(2017)$

E. Takeo, K. Inayoshi, K. Ohsuga, et al., Rapid growth of black holes accompanied with hot or warm outflows exposed to anisotropic superEddington radiation. MNRAS 476, 673-682 (2018)

J.C. Tan, C.F. McKee, The Formation of the First Stars. I. Mass Infall Rates, Accretion Disk Structure, and Protostellar Evolution. ApJ 603, 383-400 (2004)

A. Toomre, On the gravitational stability of a disk of stars. ApJ 139, 1217-1238 (1964)

R.H.D. Townsend, S.P. Owocki, D. Groote, The Rigidly Rotating Magnetosphere of $\sigma$ Orionis E. ApJ 630, 81-84 (2005)

D. Tseliakhovich, C. Hirata, Relative velocity of dark matter and baryonic fluids and the formation of the first structures. Phys. Rev. D 82(8), 083520 (2010)

M.J. Turk, T. Abel, B. O'Shea, The Formation of Population III Binaries from Cosmological Initial Conditions. Science 325, 601 (2009)

M.J. Turk, B.D. Smith, J.S. Oishi, et al., yt: A Multi-code Analysis Toolkit for Astrophysical Simulation Data. ApJS 192, 9 (2011)

M.J. Turk, J.S. Oishi, T. Abel, et al., Magnetic Fields in Population III Star Formation. ApJ 745, 154 (2012)

H. Uchida, M. Shibata, T. Yoshida, et al., Gravitational collapse of rotating supermassive stars including nuclear burning effects. Phys. Rev. D 96(8), 083016 (2017)

H. Umeda, T. Hosokawa, K. Omukai, et al., The Final Fates of Accreting Supermassive Stars. ApJ 830, 34 (2016)

W. Unno, Physical State and Stability of Super-Massive Objects. PASJ 23, $123(1971)$

E. Visbal, R. Barkana, A. Fialkov, et al., The signature of the first stars in atomic hydrogen at redshift 20. Nature 487, 70-73 (2012)

M. Volonteri, G. Lodato, P. Natarajan, The evolution of massive black hole 
seeds. MNRAS 383, 1079-1088 (2008)

$\mathrm{H}$. von Zeipel, The radiative equilibrium of a rotating system of gaseous masses. MNRAS 84, 665-683 (1924)

M. Walther, J. Oñorbe, J.F. Hennawi, et al., New Constraints on IGM Thermal Evolution from the Ly $\alpha$ Forest Power Spectrum. ApJ 872(1), $13(2019)$

D. Whalen, T. Abel, M.L. Norman, Radiation Hydrodynamic Evolution of Primordial H II Regions. ApJ 610, 14-22 (2004)

A. Whitworth, D. Summers, Self-similar condensation of spherically symmetric self-gravitating isothermal gas clouds. MNRAS 214, 1-25 (1985)

L.M. Widrow, D. Ryu, D.R.G. Schleicher, et al., The First Magnetic Fields. Space Sci. Rev. 166, 37-70 (2012)

J.H. Wise, M.J. Turk, T. Abel, Resolving the Formation of Protogalaxies. II. Central Gravitational Collapse. ApJ 682, 745-757 (2008)

J.H. Wise, T. Abel, M.J. Turk, et al., The birth of a galaxy - II. The role of radiation pressure. MNRAS 427, 311-326 (2012a)

J.H. Wise, M.J. Turk, M.L. Norman, et al., The Birth of a Galaxy: Primordial Metal Enrichment and Stellar Populations. ApJ 745, 50 (2012b)

J.H. Wise, J.A. Regan, B.W. O'Shea, et al., Formation of massive black holes in rapidly growing pre-galactic gas clouds. arXiv e-prints (2019)

K.M.J. Wollenberg, S.C.O. Glover, P.C. Clark, et al., Formation sites of Population III star formation: The effects of different levels of rotation and turbulence on the fragmentation behaviour of primordial gas. MNRAS , 287 (2020)

T.E. Woods, A. Heger, D.J. Whalen, et al., On the Maximum Mass of Accreting Primordial Supermassive Stars. ApJ 842, 6 (2017)

T.E. Woods, B. Agarwal, V. Bromm, et al., Titans of the early Universe: The Prato statement on the origin of the first supermassive black holes. PASA 36, 027 (2019)

X.-B. Wu, F. Wang, X. Fan, et al., An ultraluminous quasar with a twelvebillion-solar-mass black hole at redshift 6.30. Nature 518, 512-515 (2015)

S.-C. Yoon, F. Iocco, S. Akiyama, Evolution of the First Stars with Dark Matter Burning. ApJ 688, 1 (2008)

H.W. Yorke, C. Sonnhalter, On the Formation of Massive Stars. ApJ 569, 846-862 (2002)

N. Yoshida, K. Omukai, L. Hernquist, Protostar Formation in the Early Universe. Science 321, 669 (2008)

N. Yoshida, T. Abel, L. Hernquist, et al., Simulations of Early Structure Formation: Primordial Gas Clouds. ApJ 592, 645-663 (2003)

N. Yoshida, K. Omukai, L. Hernquist, et al., Formation of Primordial Stars in a $\Lambda$ CDM Universe. ApJ 652, 6-25 (2006)

N. Yoshida, S.P. Oh, T. Kitayama, et al., Early Cosmological H II/He III Regions and Their Impact on Second-Generation Star Formation. ApJ 663, 687-707 (2007)

E. Zackrisson, C. Binggeli, K. Finlator, et al., The Spectral Evolution of the First Galaxies. III. Simulated James Webb Space Telescope Spectra of Reionization-epoch Galaxies with Lyman-continuum Leakage. ApJ 836, 
78 (2017)

H. Zinnecker, H.W. Yorke, Toward Understanding Massive Star Formation. ARA\&A 45, 481-563 (2007) 


\section{List of Figures}

1 Density evolution in a 120 AU region around the first protostar, showing the build-up of the protostellar disk and its eventual fragmentation. The prominent two-arm spiral structure is caused by the gravitational instability in the disk, and the resulting gravitational torques provide the main source of angular momentum transport that allows disk material to accrete onto the protostar. Eventually, as mass continues to pour onto the disk from the infalling envelope, the disk becomes so unstable that regions in the spiral arms become self-gravitating in their own right. The disk fragments and a multiple system is formed. Figure adopted from Clark et al. (2011b) . . . . . . . . . . . . . . .

2 Evolution of the stellar radius with the different accretion rates, from Hosokawa et al. (2012). The upper and lower panels present the evolution with the rates lower and higher than $6 \times 10^{-2} \mathrm{M}_{\odot} \mathrm{yr}^{-1}$. The different lines represent the accretion rates as indicated by the labels. The thin green line represents the analytic mass-radius relationship given by equation (9). The filled circles on the lines denote the epoch when the hydrogen burning initiates near the center.

3 Evolution of the stellar interior structure until the stellar mass reaches $10^{5} \mathrm{M}_{\odot}$ with the accretion rate $\dot{M}_{*}=$ $1 \mathrm{M}_{\odot} \mathrm{yr}^{-1}$, from Hosokawa et al. (2013). The black solid and dashed lines represent the radial positions of the stellar surface and mass coordinates enclosing $80 \%, 60 \%, 40 \%$, and $20 \%$ of the total mass in descending order. The white and yellow areas represent radiative and convective zones where no nuclear fusion takes place. The brown stripe and pink zones indicate the radiative and convective layers where deuterium burning occurs. The green area represents a convective core where hydrogen burning occurs. . . . . . . . . . . . .

4 Limits to hydrostatic equilibrium in the $M-\dot{M}$ plan, from Haemmerlé et al. (2019). Above the solid black line, accretion is too rapid for the star to evolve in quasi-static equilibrium. At the right-hand side of the red line, stars are GR unstable (Woods et al., 2017), see Sect 2.1.4. . . . . . . . . . .

5 Mass-limits for GR stability of accreting SMSs, as a function of the (constant) accretion rate, by Umeda et al. (2016), Woods et al. (2017) and Haemmerlé et al. (2018b). . . . .

6 Constraint from the $\Omega \Gamma$-limit on the surface rotation velocity of SMSs, from Haemmerlé et al. (2018a). . . . . . . . . . . .

7 Color-coded density maps showing the evolution of the central gaseous protogalactic disk inside a metal-free photodissociated atomic cooling halo at $z \sim 203$ (adapted from Latif et al. 2013a). From top to bottom increasingly smaller scales are shown, and the time evolution runs from left to right. . . . . . . . . . 
8 Face-on and edge-on projected gas density maps of the nuclear region of the merger at $t_{0}+5 \mathrm{kyr}\left(t_{0}\right.$ is the time corresponding to the merger of the two central cores.) showing a disk-like object with radius $5 \mathrm{pc}$ (first and second panel are edge-on and faceon, respectively), and a compact inner disk-like core less than a parsec in size (third and fourth panels are face-on and edge-on, respectively). We show one particular run from the Mayer et al. 2015 paper, RUN4, which employed a modern version of the SPH hydro solver using pressure-energy formulation (GDSPH), thermal and metal diffusion, and a Wedland kernel. The figure is adapted from Mayer and Bonoli $(2019) . \quad$. . . . . . . . . . . . . . .

9 Time evolution of the gas accretion rate at different radii from the center of the merger remnant. The accretion is computed inside cylindrical shells of inner and outer radii marked in the legend and vertical thickness 2 pc. RUN4 from Mayer et al. (2015) is used, which is also shown in Figure 3. Adapted from Mayer et al. (2015).)

10 The figure, adapted from Wise et al. (2019), shows, for their high resolution re-simulations of two rare peaks in the Renaissance cosmological simulation (indicated respectively, by the dashed and solid lines); gas temperature (c, black lines), $H_{2}$ fraction (c, green lines), radial mass infall rates (d, black lines), and radial infall velocities ( $d$, green lines). The orange circles indicate the Jeans mass and associated quantities at that mass scale when the object first becomes gravitationally unstable, whereas the red squares represent the same quantities at the end of the simulation. . . . .

11 Cosmic Reionization Fundamentals. After recombination at $z \sim$ 1100 , hydrogen in the IGM remained neutral until the first stars and galaxies formed at $z \sim 15-20$. These primordial systems released energetic UV photons capable of ionizing local bubbles of hydrogen gas. As the abundance of these early galaxies increased, $\mathrm{H}$ in bubbles overlapped and progressively larger volumes became ionized. This reionization process completed at $z \sim 6$, approximately 1 Gyr after the Big Bang. At lower redshifts, the IGM remains highly ionized through radiation provided by star-forming galaxies and the gas accretion onto supermassive black holes that powers quasars. (From Robertson et al. 2010) . . . . . . . . . . 
12 Observational Probes of Reionization: I. Left: Fraction of Lyman break galaxies that display Ly $\alpha$ in emission at an EW $25 \AA$ plotted as a function of redshift. The values at $z=7$ and 8 reflect differential measurements with the data at $z=6$. (From (Schenker et al., 2014).) Center: Evolution of the cosmic star formation rate (SFR) density at high redshift integrated down to a UV luminosity of $M_{\mathrm{UV}}=-17.0\left(\mathrm{SFR} \gtrsim 0.3 M_{\odot} \mathrm{yr}^{-1}\right)$. New measurements from the combination of all $H S T$ fields (filled dark red circle) suggest a rapid, accelerated evolution of this quantity between redshifts 8 and 10. The orange shaded region shows the relative evolution of the cumulative DM halo mass function integrated down to $\log M_{\text {halo }} / M_{\odot}=9.5-10.5$. (From (Oesch et al., 2017).) Right: Synthetic spectrum of a single $M_{*} \approx 7 \times 10^{8} M_{\odot}, m_{\mathrm{AB}}=27$, dust-free star-forming galaxy for different values of the LyC escape fraction $f_{\text {esc }}$ : 0.0 (red), 0.5 (orange), 0.7 (green) and 0.9 (blue). The spectrum has been degraded to the spectral resolution of the $J W S T$ /NIRSpec $R=100$ prism, and with noise level corresponding to a $10 \mathrm{~h}$ exposure. The $f_{\text {esc }}$ parameter mainly affects the strength of the emission lines relative to the continuum, and this effect remains detectable for the strongest nebular lines. (From Zackrisson et al. 2017).) . . . . . . . . . . . . . .

13 Observational Probes of Reionization: II. Left: Fluctuations in the $\mathrm{H}$ I ionizing background for a galaxies+AGN source model. The map shows the $\mathrm{H}$ I photoionization rate at $z=5.6$ in a slice of thickness $6.25 \mathrm{Mpc} / h$ for a UV background model where AGNs produce $50 \%$ of the global average $\mathrm{H}$ I photoionization rate. The clustering of AGNs leads to large-scale variations in the mean free path of ionizing photons that amplify fluctuations in the ionizing background. (From (D'Aloisio et al. 2017).) Center: Ly $\alpha$ transmission spikes towards the $z=7.1$ quasar ULAS J1120+0641. (From (Barnett et al., 2017).) Right: Evolution of the IGM temperature at mean density $T_{0}$ and $\rho-T$ relation slope $\gamma$ as a function of redshift. (From (Walther et al., 2019).) . . . . . . . . . . 
14 Average hydrogen neutral fraction, $\left\langle x_{\mathrm{HI}}\right\rangle$, of a multi-phase IGM. Left panel: Reionization histories predicted by integrating Equation 19 with a constant emission rate of ionizing photons per hydrogen atom, $\left\langle\dot{n}_{\gamma}\right\rangle /\left\langle n_{\mathrm{H}}\right\rangle=3$ phot $\mathrm{Gyr}^{-1}$. The data points represent constraints on the ionization state of the IGM from: the GunnPeterson optical depth at $5<z<6.1$ (red open circles, Fan et al. 2006), measurements of the Ly $\alpha$ forest opacity combined with hydrodynamical simulations (green triangles, Bolton and Haehnelt 2007 ), the dark pixel statistics at $z=5.6$ and $z=5.9$ (blue squares, McGreer et al. 2015), the gap/peak statistics at $z=6.32$ (magenta square, Gallerani et al. 2008), the damping wing absorption profiles in the spectra of quasars at $z=6.3$ (orange pentagon, Schroeder et al. 2013) and $z=7.1$ (turquoise hexagon, Greig et al. 2017), the redshift-dependent prevalence of LAEs in narrow-band surveys at $z=7$ and $z=8$ (firebrick stars, Schenker et al. 2014) and their clustering properties at $z=6.6$ (gold hexagon, Ouchi et al. 2010). The limit on the redshift at which $\left\langle x_{\mathrm{HI}}\right\rangle=0.5$, extracted from Planck's CMB analysis (Planck Collaboration et al., 2016b), is plotted as the green dot. Right panel: Reionization histories in three radiation-hydrodynamic simulations of the Reionization of Cosmic Hydrogen (SCORCH) project (Doussot et al. 2017 ) that have different functional forms for the luminosity-weighted escape fraction $f_{\mathrm{esc}}$. . . . . . . . . . . . . . . . . 


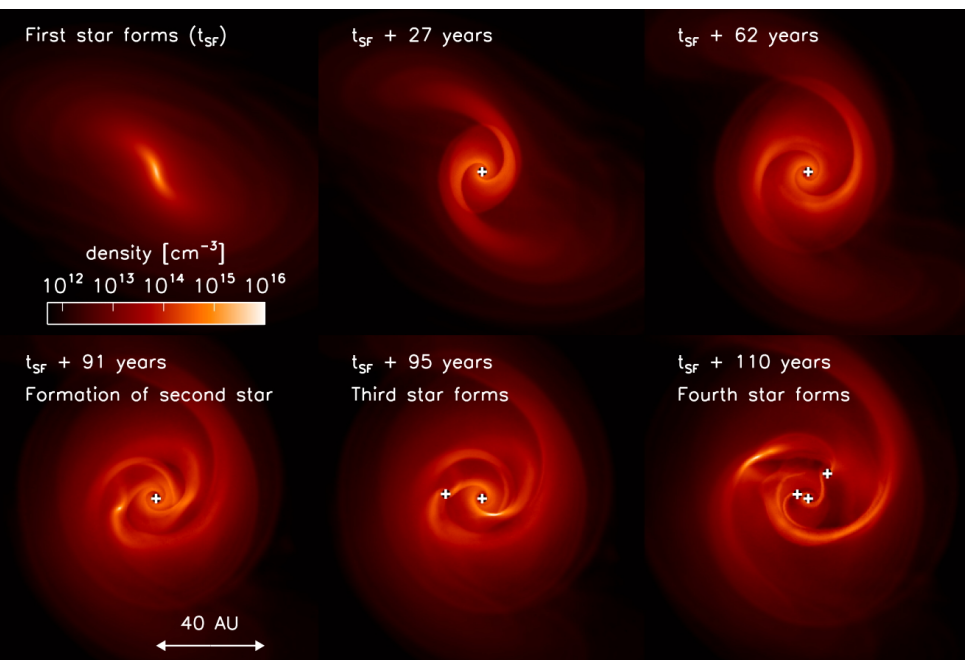

Fig. 1 Density evolution in a 120 AU region around the first protostar, showing the build-up of the protostellar disk and its eventual fragmentation. The prominent two-arm spiral structure is caused by the gravitational instability in the disk, and the resulting gravitational torques provide the main source of angular momentum transport that allows disk material to accrete onto the protostar. Eventually, as mass continues to pour onto the disk from the infalling envelope, the disk becomes so unstable that regions in the spiral arms become self-gravitating in their own right. The disk fragments and a multiple system is formed. Figure adopted from Clark et al. (2011b). 


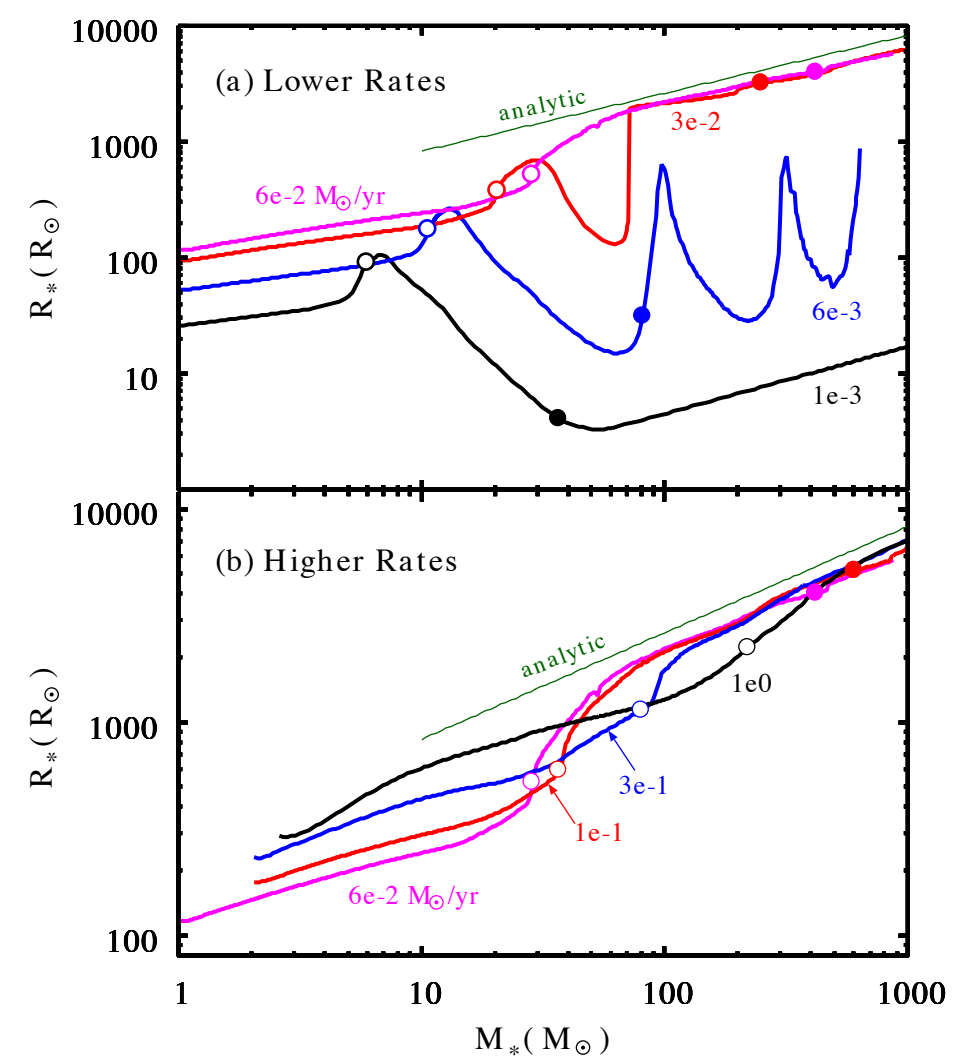

Fig. 2 Evolution of the stellar radius with the different accretion rates, from Hosokawa et al. (2012). The upper and lower panels present the evolution with the rates lower and higher than $6 \times 10^{-2} \mathrm{M}_{\odot} \mathrm{yr}^{-1}$. The different lines represent the accretion rates as indicated by the labels. The thin green line represents the analytic mass-radius relationship given by equation (9). The filled circles on the lines denote the epoch when the hydrogen burning initiates near the center. 


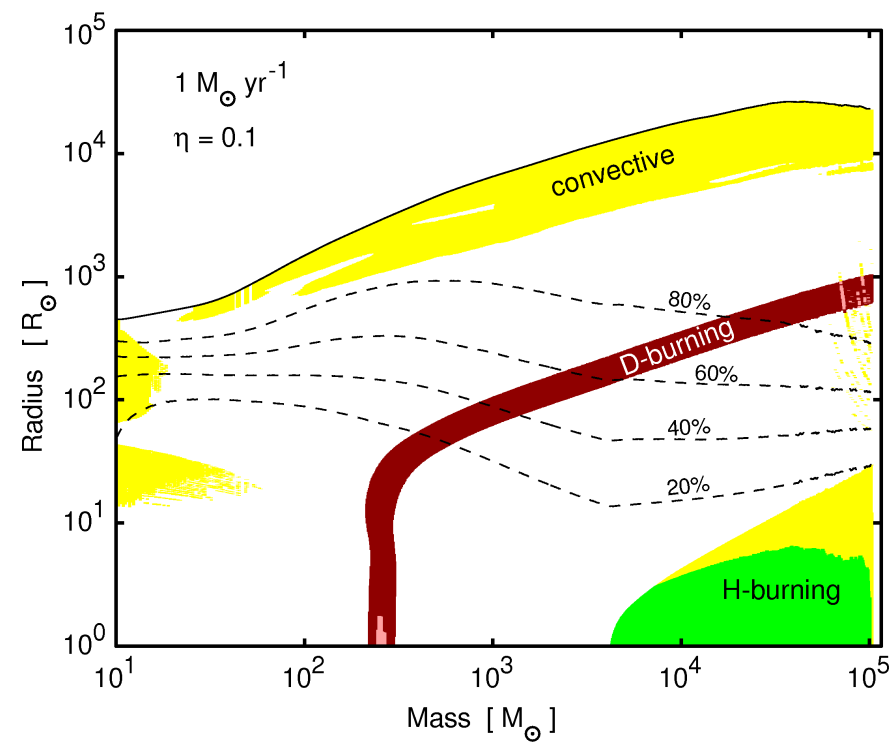

Fig. 3 Evolution of the stellar interior structure until the stellar mass reaches $10^{5} \mathrm{M}_{\odot}$ with the accretion rate $\dot{M}_{*}=1 \mathrm{M}_{\odot} \mathrm{yr}^{-1}$, from Hosokawa et al. (2013). The black solid and dashed lines represent the radial positions of the stellar surface and mass coordinates enclosing $80 \%, 60 \%, 40 \%$, and $20 \%$ of the total mass in descending order. The white and yellow areas represent radiative and convective zones where no nuclear fusion takes place. The brown stripe and pink zones indicate the radiative and convective layers where deuterium burning occurs. The green area represents a convective core where hydrogen burning occurs. 


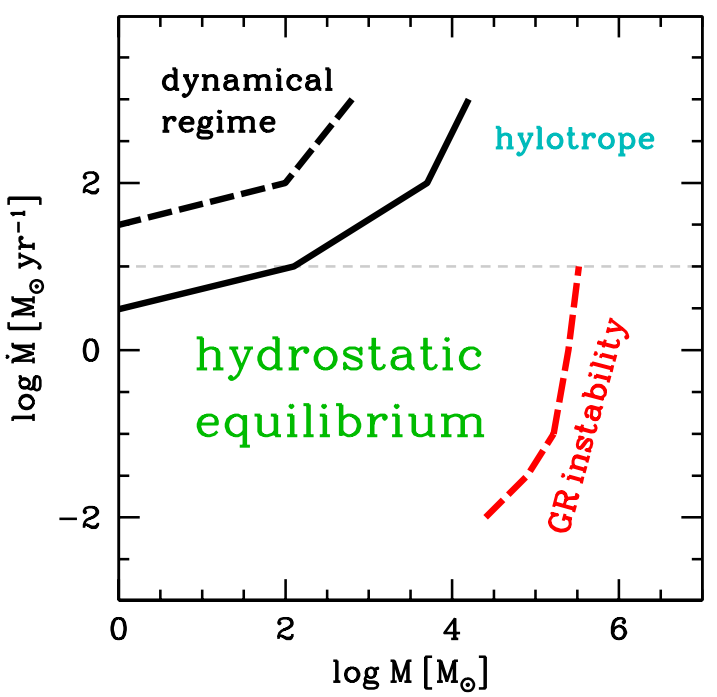

Fig. 4 Limits to hydrostatic equilibrium in the $M-\dot{M}$ plan, from Haemmerle et al. (2019). Above the solid black line, accretion is too rapid for the star to evolve in quasi-static equilibrium. At the right-hand side of the red line, stars are GR unstable (Woods et al. 2017), see Sect 2.1.4. 


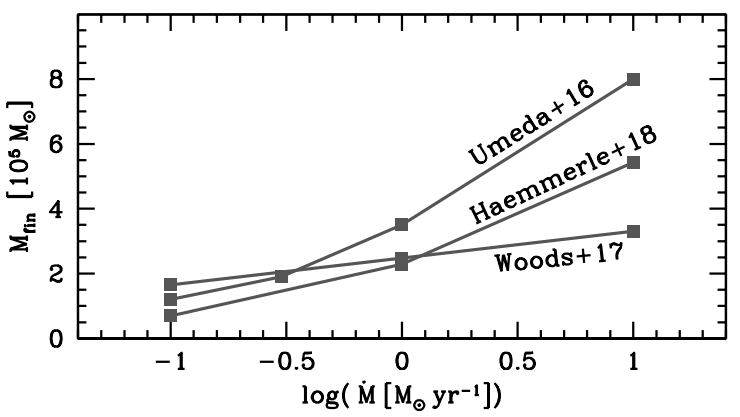

Fig. 5 Mass-limits for GR stability of accreting SMSs, as a function of the (constant) accretion rate, by Umeda et al. (2016), Woods et al. (2017) and Haemmerlé et al. (2018b). 


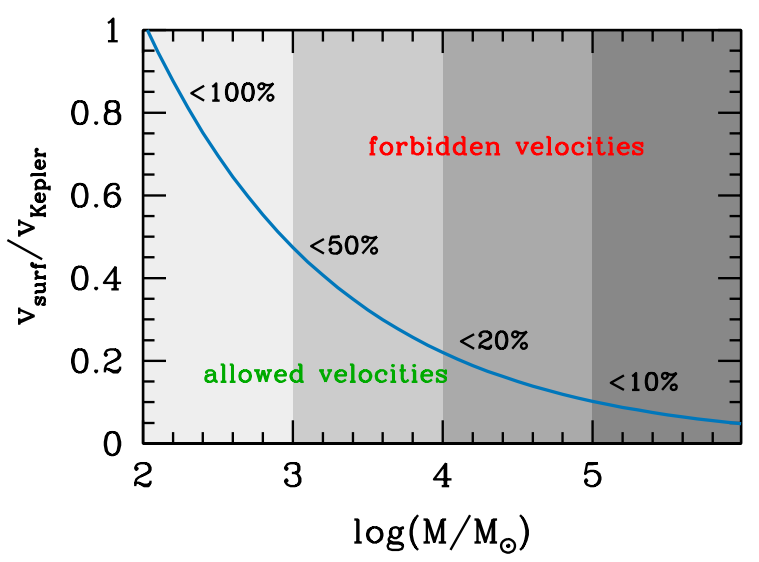

Fig. 6 Constraint from the $\Omega \Gamma$-limit on the surface rotation velocity of SMSs, from Haemmerlé et al. (2018a). 

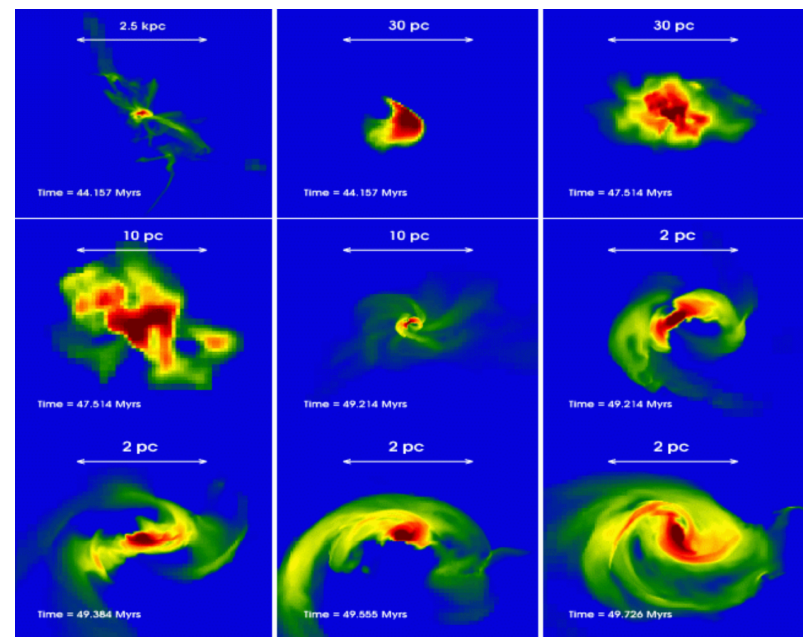

Fig. 7 Color-coded density maps showing the evolution of the central gaseous protogalactic disk inside a metal-free photodissociated atomic cooling halo at $z \sim 203$ (adapted from Latif et al. 2013a). From top to bottom increasingly smaller scales are shown, and the time evolution runs from left to right. 


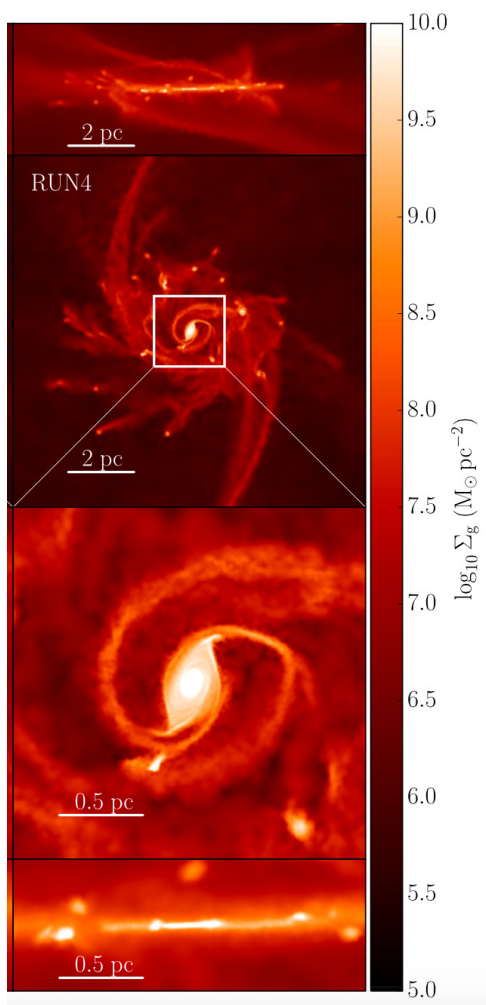

Fig. 8 Face-on and edge-on projected gas density maps of the nuclear region of the merger at $t_{0}+5 \mathrm{kyr}$ ( $t_{0}$ is the time corresponding to the merger of the two central cores.) showing a disk-like object with radius $5 \mathrm{pc}$ (first and second panel are edgeon and face-on, respectively), and a compact inner disk-like core less than a parsec in size (third and fourth panels are face-on and edge-on, respectively). We show one particular run from the Mayer et al. 2015 paper, RUN4, which employed a modern version of the SPH hydro solver using pressure-energy formulation (GDSPH), thermal and metal diffusion, and a Wedland kernel. The figure is adapted from Mayer and Bonoli (2019). 


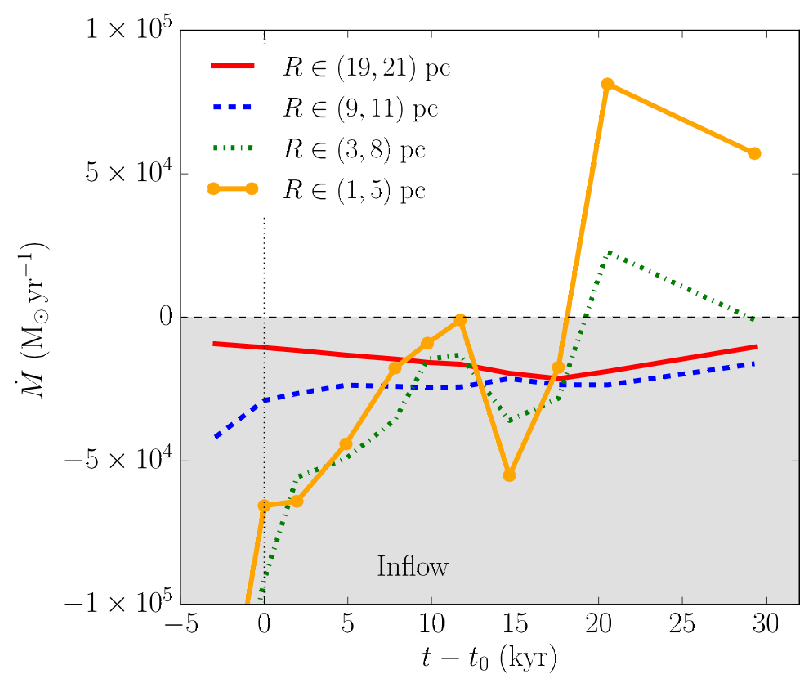

Fig. 9 Time evolution of the gas accretion rate at different radii from the center of the merger remnant. The accretion is computed inside cylindrical shells of inner and outer radii marked in the legend and vertical thickness 2 pc. RUN4 from Mayer et al. (2015) is used, which is also shown in Figure 3. Adapted from Mayer et al. (2015).) 


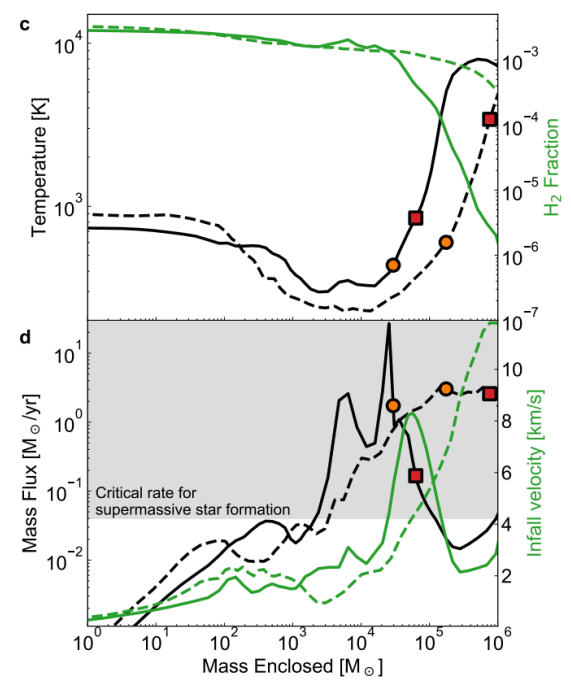

Fig. 10 The figure, adapted from Wise et al. (2019), shows, for their high resolution re-simulations of two rare peaks in the Renaissance cosmological simulation (indicated respectively, by the dashed and solid lines); gas temperature (c, black lines), $\mathrm{H}_{2}$ fraction (c, green lines), radial mass infall rates (d, black lines), and radial infall velocities (d, green lines). The orange circles indicate the Jeans mass and associated quantities at that mass scale when the object first becomes gravitationally unstable, whereas the red squares represent the same quantities at the end of the simulation. 


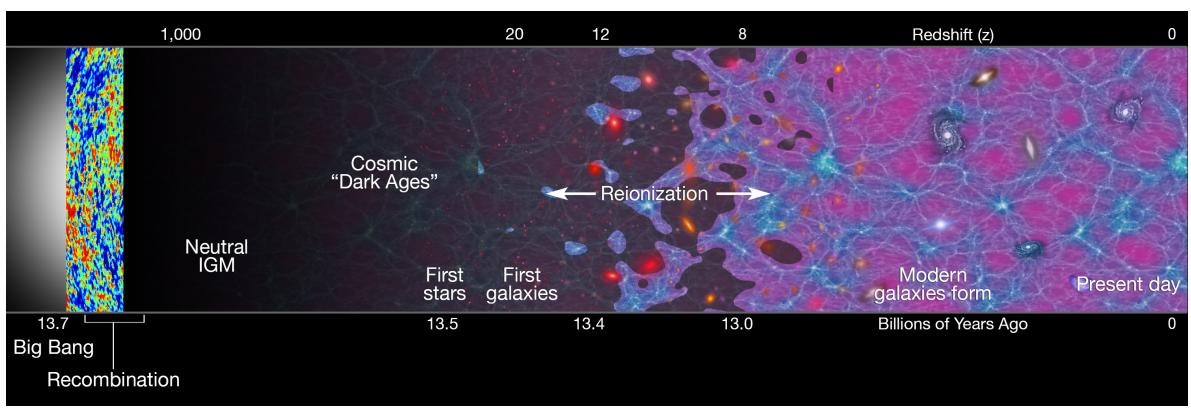

Fig. 11 Cosmic Reionization Fundamentals. After recombination at $z \sim 1100$, hydrogen in the IGM remained neutral until the first stars and galaxies formed at $z \sim 15-20$. These primordial systems released energetic UV photons capable of ionizing local bubbles of hydrogen gas. As the abundance of these early galaxies increased, $\mathrm{H}$ II bubbles overlapped and progressively larger volumes became ionized. This reionization process completed at $z \sim 6$, approximately 1 Gyr after the Big Bang. At lower redshifts, the IGM remains highly ionized through radiation provided by star-forming galaxies and the gas accretion onto supermassive black holes that powers quasars. (From Robertson et al. 2010) 

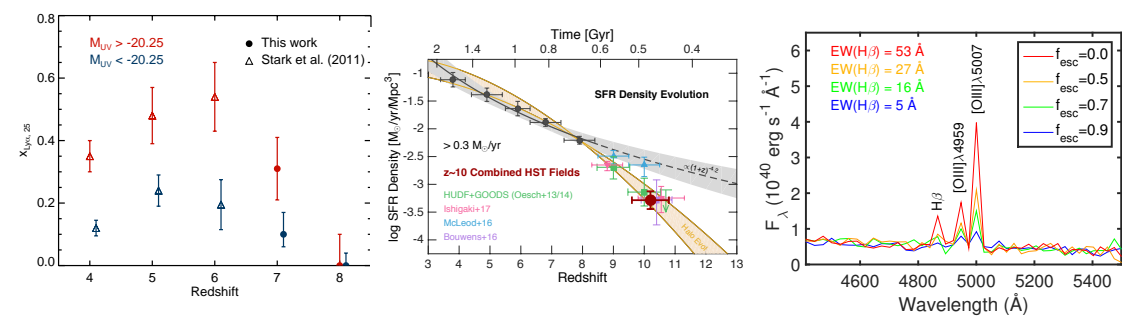

Fig. 12 Observational Probes of Reionization: I. Left: Fraction of Lyman break galaxies that display Ly $\alpha$ in emission at an EW $25 \AA$ plotted as a function of redshift. The values at $z=7$ and 8 reflect differential measurements with the data at $z=6$. (From (Schenker et al. 2014).) Center: Evolution of the cosmic star formation rate (SFR) density at high redshift integrated down to a UV luminosity of $M_{\mathrm{UV}}=-17.0$ (SFR $\left.\gtrsim 0.3 M_{\odot} \mathrm{yr}^{-1}\right)$. New measurements from the combination of all HST fields (filled dark red circle) suggest a rapid, accelerated evolution of this quantity between redshifts 8 and 10. The orange shaded region shows the relative evolution of the cumulative DM halo mass function integrated down to $\log M_{\text {halo }} / M_{\odot}=9.5-10.5$. (From (Oesch et al., 2017).) Right: Synthetic spectrum of a single $M_{*} \approx 7 \times 10^{8} M_{\odot}$, $m_{\mathrm{AB}}=27$, dust-tree star-forming galaxy for different values of the LyC escape fraction $f_{\text {esc }}$ : 0.0 (red), 0.5 (orange), 0.7 (green) and 0.9 (blue). The spectrum has been degraded to the spectral resolution of the JWST/NIRSpec $R=100$ prism, and with noise level corresponding to a $10 \mathrm{~h}$ exposure. The $f_{\text {esc }}$ parameter mainly affects the strength of the emission lines relative to the continuum, and this effect remains detectable for the strongest nebular lines. (From (Zackrisson et al., 2017).) 

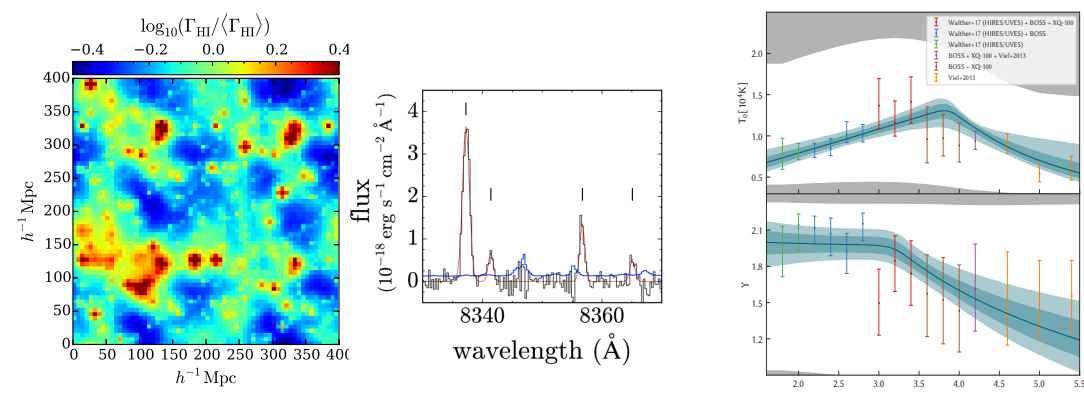

Fig. 13 Observational Probes of Reionization: II. Left: Fluctuations in the $\mathrm{H}$ ionizing background for a galaxies+AGN source model. The map shows the $\mathrm{H}$ I photoionization rate at $z=5.6$ in a slice of thickness $6.25 \mathrm{Mpc} / h$ for a UV background model where AGNs produce $50 \%$ of the global average $\mathrm{H} \mathrm{I}$ photoionization rate. The clustering of AGNs leads to large-scale variations in the mean free path of ionizing photons that amplify fluctuations in the ionizing background. (From (D'Aloisio et al., 2017).) Center: Ly $\alpha$ transmission spikes towards the $z=7.1$ quasar ULAS J1120+0641. (From (Barnett et al., 2017).)

Right: Evolution of the IGM temperature at mean density $T_{0}$ and $\rho-T$ relation slope $\gamma$ as a function of redshift. (From (Walther et al., 2019).) 

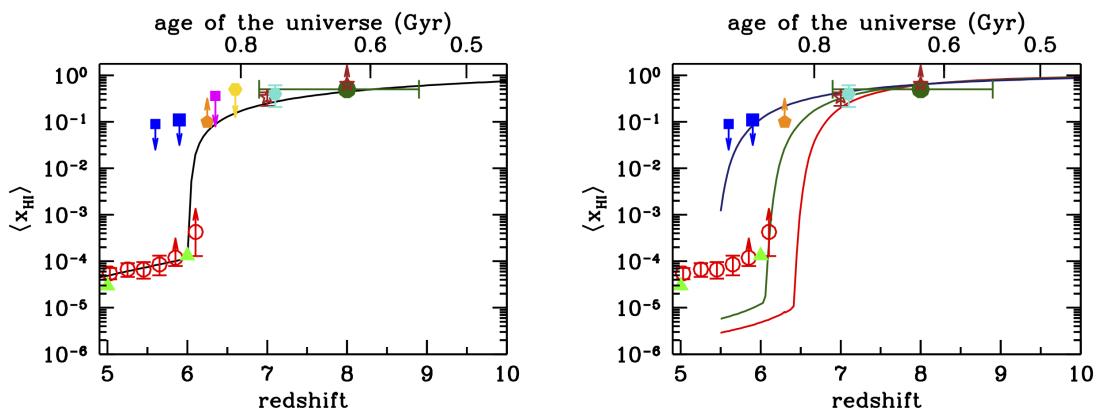

Fig. 14 Average hydrogen neutral fraction, $\left\langle x_{\mathrm{HI}}\right\rangle$, of a multi-phase IGM. Left panel: Reionization histories predicted by integrating Equation 190 with a constant emission rate of ionizing photons per hydrogen atom, $\left\langle\dot{n}_{\gamma}\right\rangle /\left\langle n_{\mathrm{H}}\right\rangle=3$ phot $\mathrm{Gyr}^{-1}$. The data points represent constraints on the ionization state of the IGM from: the Gunn-Peterson optical depth at $5<z<6.1$ (red open circles, Fan et al. 2006), measurements of the Ly $\alpha$ forest opacity combined with hydrodynamical simulations (green triangles, Bolton and Haehnelt 2007), the dark pixel statistics at $z=5.6$ and $z=5.9$ (blue squares, McGreer et al. 2015), the gap/peak statistics at $z=6.32$ (magenta square, Gallerani et al. 2008), the damping wing absorption profiles in the spectra of quasars at $z=6.3$ (orange pentagon, Schroeder et al. 2013) and $z=7.1$ (turquoise hexagon, Greig et al. 2017), the redshift-dependent prevalence of LAEs in narrow-band surveys at $z=7$ and $z=8$ (firebrick stars, Schenker et al. 2014) and their clustering properties at $z=6.6$ (gold hexagon, Ouchi et al. 2010). The limit on the redshift at which $\left\langle x_{\mathrm{HI}}\right\rangle=0.5$, extracted from Planck's CMB analysis (Planck Collaboration et al., 2016b), is plotted as the green dot. Right panel: Reionization histories in three radiation-hydrodynamic simulations of the Reionization of Cosmic Hydrogen (SCORCH) project (Doussot et al. 2017) that have different functional forms for the luminosity-weighted escape fraction $f_{\text {esc }}$. 